\title{
Use of real Dirac matrices in two-dimensional coupled linear optics
}

\author{
C. Baumgarten* \\ Paul Scherrer Institute, 5232 Villigen PSI, Switzerland \\ (Received 12 September 2011; published 18 November 2011)
}

\begin{abstract}
The Courant-Snyder theory for two-dimensional coupled linear optics is presented, based on the systematic use of the real representation of the Dirac matrices. Since any real $4 \times 4$ matrix can be expressed as a linear combination of these matrices, the presented ansatz allows for a comprehensive and complete treatment of two-dimensional linear coupling. A survey of symplectic transformations in two dimensions is presented. A subset of these transformations is shown to be identical to rotations and Lorentz boosts in Minkowski space-time. The transformation properties of the classical state vector are formulated and found to be analog to those of a Dirac spinor. The equations of motion for a relativistic charged particle - the Lorentz force equations - are shown to be isomorph to envelope equations of twodimensional linear coupled optics. A universal and straightforward method to decouple two-dimensional harmonic oscillators with constant coefficients by symplectic transformations is presented, which is based on this isomorphism. The method yields the eigenvalues (i.e., tunes) and eigenvectors and can be applied to a one-turn transfer matrix or directly to the coefficient matrix of the linear differential equation.
\end{abstract}

DOI: 10.1103/PhysRevSTAB.14.114002

\section{INTRODUCTION}

Even though there is continuous interest in this field (see, for instance, [1-11]), the treatment of coupled linear optics in two (or more) degrees of freedom has not yet reached the same level of generality, transparency, and conceptual clarity as provided by the Courant-Synder theory for one degree of freedom.

This article is about coupled linear optics as required to describe, for instance, the motion of charged particles in accelerators and ion beam optics. Even though ion beam optics is in principle three dimensional, often symmetries can be used to reduce the problem to the treatment of twodimensional systems. In accelerators like cyclotrons or synchrotrons, the beam circulates in the horizontal plane and the electric and magnetic fields are symmetric with respect to this so-called median plane. In this case vertical motion is neither coupled to the horizontal nor to the longitudinal motion. But the dispersion of the bending magnets couples horizontal and longitudinal motion. In other configurations, the transversal degrees of freedom are coupled with each other by solenoid magnets, but not with the longitudinal motion-or the longitudinal degree of freedom is "hidden" as the beam is not bunched but continuous. Therefore we will treat coupling in two dimensions like most authors [1-10]. In an accompanying paper, the problem of transverse-longitudinal coupling by space charge forces in isochronous cyclotrons is treated in linear

\footnotetext{
*christian.baumgarten@psi.ch
}

Published by the American Physical Society under the terms of the Creative Commons Attribution 3.0 License. Further distribution of this work must maintain attribution to the author(s) and the published article's title, journal citation, and DOI.
PACS numbers: 47.10.Df, 41.75.-i, 05.45.Xt, 03.30.+p

approximation [12]. This special type of coupling does not allow to apply the method of Teng and Edwards without modifications [12]. Other authors like Qin and Davidson restrict themselves to special forms of the Hamilton function so that their treatment lacks generality [3].

The real Dirac matrices (RDMs) have been known for a long time as the Majorana representation, going back to a paper by Ettore Majorana [13,14]. In this article, we will use the RDMs in a very practical way that has-to the knowledge of the author-not yet attracted much attention. The RDMs form a basis of $4 \times 4$ matrices with remarkable properties also - and maybe especially —in the context of classical mechanics. The RDMs are essential ingredients for a formulation of a theory of symplectic flow in two dimensions. They enable one to survey all possible symplectic transformations in an elegant and straight manner.

The use of real instead of the complex Dirac matrices has several reasons: First, linear coupled optics is a classical theory and the relevant terms in the Hamilton function are real. Second, the RDMs are a complete basis: Any real $4 \times 4$ matrix can be written as a linear combination of the RDMs. This in fact guarantees generality and completeness of the presented theory of linear coupled motion in two dimensions. Finally, the RDMs are discriminable with respect to all relevant structural properties: Each matrix is either symplectic or antisymplectic, either even or odd, two RDMs either commute or anticommute. The use of the RDMs supports another clear distinction-real or imaginary.

Furthermore, the introduction of RDMs into classical mechanics may provide new insights into the relationship of Hamiltonian mechanics, special relativity, and the Dirac equation. It has been known for a long time that the mathematical formalism of Twiss parameters and 
Courant-Snyder invariants can be applied to quantum systems [15]. But no attempt has yet been made to apply the tools of quantum mechanics in classical mechanics.

Nevertheless we emphasize that a lot of the presented formalism, i.e., practically all equations that do not refer to other matrices than $\gamma_{0}$, may be applied in arbitrary dimensions, if $\gamma_{0}$ is extended correspondingly. This holds especially for the concept of the symplex, which we introduce to identify the components of the "force matrix." We will demonstrate the significance of the RDMs for the treatment of coupled linear motion, in the context of transfer matrices and eigensystems. This leads in a natural way to the twodimensional generalization of the Courant-Snyder theory. In the second part, we demonstrate how the RDMs can be used in the context of the second moments using Poisson brackets. We show that both, the Maxwell equations and the Lorentz force equations, can be formulated casually in terms of the RDMs. This brings up an analogy which we call the "electromechanical equivalence." Effectively we use the isomorphism of symplectic transformations with Lorentz boosts and rotations in Minkowski space to introduce a physical nomenclature of symplectic transformations in two dimensions. Based on this equivalence and on the distinction of even and odd matrices, we finally develop a general algorithm that allows one to determine the symplectic decoupling transformation. Furthermore, the algorithm enables one to compute the eigenvalues and the eigenvectors of stable two-dimensional symplectic systems.

\section{REAL DIRAC MATRICES IN COUPLED LINEAR OPTICS}

The position and direction of a charged particle within a beam is usually described by its coordinates and angles relative to the reference trajectory. In case of two transversal coordinates, the position of the particle is described by $x, x^{\prime}, y, y^{\prime}$, where $x$ is the horizontal and $y$ the vertical ("axial") coordinate. The dashes represent the derivative with respect to the path length $s$ along the reference orbit. In case of transverse-longitudinal coupling, a common choice of the coordinates is $x, x^{\prime}, l, \delta$, where $l$ is the longitudinal coordinate with respect to the bunch center and $\delta=\frac{p-p_{0}}{p_{0}}$ is the relative momentum deviation, where $p$ is the momentum of a specific ion and $p_{0}$ is the average momentum.

Since the formalism is related to classical Hamiltonian mechanics, we prefer to write $q_{i}$ and $p_{i}$ for the dynamical variables and we collect them in a vector $\psi$ :

$$
\psi=\left(q_{1}, p_{1}, q_{2}, p_{2}\right)^{T} .
$$

We could as well change the ordering of the variables and use, for example,

$$
\psi=\left(q_{1}, q_{2}, p_{1}, p_{2}\right)^{T} .
$$

This may (of course) have no influence on the physical situation and its description, but it leads to a different ordering of the RDMs. Four elements can be ordered in $4 !=24$ different ways, but since we do not distinguish the indices, there are $4 ! / 2=12$ different permutations of the elements in $\psi$ and corresponding systems of RDMs as listed in Table III.

We identify the Dirac matrix $\gamma_{0}$ with the symplectic unit matrix, which is usually denoted by $I, J$, or $S$. In the case of Eq. (1), the "time direction" $\gamma_{0}$ is

$$
\gamma_{0}=\left(\begin{array}{cccc}
0 & 1 & 0 & 0 \\
-1 & 0 & 0 & 0 \\
0 & 0 & 0 & 1 \\
0 & 0 & -1 & 0
\end{array}\right)
$$

in the case of Eq. (2), the form is

$$
\eta_{0}=\left(\begin{array}{cccc}
0 & 0 & 1 & 0 \\
0 & 0 & 0 & 1 \\
-1 & 0 & 0 & 0 \\
0 & -1 & 0 & 0
\end{array}\right) .
$$

$\gamma_{0}$ is a skew-symmetric matrix that squares to the negative unit matrix $\gamma_{0}^{2}=-\mathbf{1}$, while in the usual definition of the Dirac matrices one has $\gamma_{0}^{2}=\mathbf{1}$. The other three basic matrices $\gamma_{1} \ldots \gamma_{3}$ are defined by the requirement that they must anticommute with $\gamma_{0}$ and with each other and that they square to the opposite sign of $\gamma_{0}^{2}$. The signature of the metric tensor $g_{\mu \nu}$, hence, is $(-1,+1,+1,+1)-$ instead of $(+1,-1,-1,-1)[16,17]$ :

$$
g_{\mu \nu}=\operatorname{Diag}(-1,1,1,1)=\frac{\gamma_{\mu} \gamma_{\nu}+\gamma_{\nu} \gamma_{\mu}}{2}
$$

The other matrices that we use to form the symplectic basis are

$$
\begin{gathered}
\gamma_{1}=\left(\begin{array}{cccc}
0 & -1 & 0 & 0 \\
-1 & 0 & 0 & 0 \\
0 & 0 & 0 & 1 \\
0 & 0 & 1 & 0
\end{array}\right) \\
\gamma_{2}=\left(\begin{array}{llll}
0 & 0 & 0 & 1 \\
0 & 0 & 1 & 0 \\
0 & 1 & 0 & 0 \\
1 & 0 & 0 & 0
\end{array}\right)
\end{gathered}
$$

$$
\gamma_{3}=\left(\begin{array}{cccc}
-1 & 0 & 0 & 0 \\
0 & 1 & 0 & 0 \\
0 & 0 & -1 & 0 \\
0 & 0 & 0 & 1
\end{array}\right)
$$

The remaining matrices are defined by 


$$
\begin{array}{rlrl}
\gamma_{14} & =\gamma_{0} \gamma_{1} \gamma_{2} \gamma_{3} ; & \gamma_{15}=\mathbf{1} \\
\gamma_{4}=\gamma_{0} \gamma_{1} ; & \gamma_{7}=\gamma_{14} \gamma_{0} \gamma_{1}=\gamma_{2} \gamma_{3} \\
\gamma_{5}=\gamma_{0} \gamma_{2} ; & \gamma_{8}=\gamma_{14} \gamma_{0} \gamma_{2}=\gamma_{3} \gamma_{1} \\
\gamma_{6}=\gamma_{0} \gamma_{3} ; & \gamma_{9}=\gamma_{14} \gamma_{0} \gamma_{3}=\gamma_{1} \gamma_{2} \\
\gamma_{10}=\gamma_{14} \gamma_{0}=\gamma_{1} \gamma_{2} \gamma_{3} & \\
\gamma_{11}=\gamma_{14} \gamma_{1}=\gamma_{0} \gamma_{2} \gamma_{3} & \\
\gamma_{12}=\gamma_{14} \gamma_{2}=\gamma_{0} \gamma_{3} \gamma_{1} & \\
\gamma_{13} & =\gamma_{14} \gamma_{3}=\gamma_{0} \gamma_{1} \gamma_{2} .
\end{array}
$$

Note that the definition deviates from the conventions in particle physics, where the product $\gamma_{0} \gamma_{1} \gamma_{2} \gamma_{3}$ is usually labeled $\gamma_{5}$. The matrices are explicitly given in Appendix B. If $\mathbf{A}$ is an arbitrary real-valued $4 \times 4$ matrix, then $\mathbf{A}$ can be written as a linear combination of RDMs:

$$
\mathbf{A}=\sum_{k=0}^{15} a_{k} \gamma_{k}
$$

where the RDM coefficients $a_{k}$ are given by the scalar product

$$
a_{k}=\mathbf{A} \cdot \gamma_{k}=\frac{\left(\gamma_{k}\right)^{2}}{4} \operatorname{Tr}\left(\frac{\mathbf{A} \gamma_{k}+\gamma_{k} \mathbf{A}}{2}\right),
$$

and $\operatorname{Tr}(\mathbf{X})$ is the trace of $\mathbf{X}$. The RDMs form a group and are the basis of a vector space. The associated algebra is the real Clifford algebra $\mathrm{Cl}(3,1)[16,17]$.

\section{A. The Hamiltonian}

Linear coupled optics is characterized by a Hamiltonian function of the classical harmonic oscillator form

$$
H=\frac{1}{2} \psi^{T} \mathbf{A} \psi,
$$

where the superscript " $T$ " denotes a transposed vector or matrix. A is a (generally time-dependent) symmetric matrix. The Hamilton equations of motion (EQOM) have the familiar form

$$
\dot{q}_{i}=\frac{\partial H}{\partial p_{i}} \quad \dot{p}_{i}=-\frac{\partial H}{\partial q_{i}},
$$

or, in vector notation,

$$
\dot{\psi}=\gamma_{0} \nabla_{\psi} H,
$$

where the dot represents the time derivative. It is well known that the Jacobian matrix $\mathbf{M}$ of a canonical transformation is symplectic, i.e., that [18]

$$
\mathbf{M} \gamma_{0} \mathbf{M}^{T}=\gamma_{0} \text {. }
$$

A matrix $\mathbf{M}$ is antisymplectic, if

$$
\mathbf{M} \gamma_{0} \mathbf{M}^{T}=-\gamma_{0} \text {. }
$$

Equation (15) includes also

$$
\begin{gathered}
\mathbf{M}^{T} \gamma_{0} \mathbf{M}=\gamma_{0}, \quad \mathbf{M}^{T}=-\gamma_{0} \mathbf{M}^{-1} \gamma_{0}, \\
\mathbf{M}^{-1}=-\gamma_{0} \mathbf{M}^{T} \gamma_{0} .
\end{gathered}
$$

The product of symplectic matrices is symplectic. But note: Also the product of two antisymplectic matrices is symplectic (see also Table I).

\section{B. The force matrix and the definition of a symplex}

From Eqs. (12) and (14), one derives the following linear EQOM:

$$
\dot{\psi}=\gamma_{0} \mathbf{A} \psi=\mathbf{F} \psi,
$$

where $\mathbf{F}$ is the (generally time-dependent) force matrix.

The antisymmetry of $\gamma_{0}$ and the symmetry of $\mathbf{A}$ yield

$$
\mathbf{F}^{T}=\left(\gamma_{0} \mathbf{A}\right)^{T}=\mathbf{A}^{T} \gamma_{0}^{T}=-\mathbf{A} \gamma_{0}=\gamma_{0} \gamma_{0} \mathbf{A} \gamma_{0}=\gamma_{0} \mathbf{F} \gamma_{0}
$$

In the following we call a matrix $\mathbf{F}$ that fulfills Eq. (19) a symplex (not "simplex"). Symplices are sometimes called "infinitesimally symplectic" or "Hamiltonian" [19], but

TABLE I. Properties of the real $\gamma$ matrices. The type encoding is $V$ for vectors, $B$ for bivector, $A$ for axial vector, $P$ for pseudoscalar, and $S$ for scalar and refers to the symplectic transformation properties. The subscripts $t(s)$ indicate time(space)like components of a 4-vector, respectively. The column labeled $\gamma_{a}$ gives the sign $s$ that fulfills the following equation: $\gamma_{a} \gamma_{x} \gamma_{a}=s \gamma_{x}$. The column labeled "s/a" indicates whether $\gamma_{x}$ is a symplex or an antisymplex, respectively. Accordingly, the column labeled "S/A" tells if $\gamma_{x}$ is symplectic or antisymplectic, respectively. The column labeled "e/o" indicates whether the corresponding $\gamma$ matrix is even or odd. Even matrices are nonzero only in the block-diagonal components, odd matrices are zero in the block-diagonal components. Finally, the column labeled $\tilde{\gamma}_{0}$ denotes if a basis exists such that $\gamma_{x}$ appears as the "time direction," i.e., if a basis exists in which $\gamma_{x}$ plays the role of $\gamma_{0}$. This is only the case for antisymmetric matrices, which are either both a symplex and symplectic or none of it.

\begin{tabular}{lccccccccccc}
\hline \hline Type & $\gamma_{x}$ & Electromagnetic & $\gamma_{x}^{2}$ & $\gamma_{x}^{T}$ & $\gamma_{0}$ & $\gamma_{14}$ & $\gamma_{10}$ & $\mathrm{~s} / \mathrm{a}$ & $\mathrm{S} / \mathrm{A}$ & $\tilde{\gamma}_{0}$ & $\mathrm{e} / \mathrm{o}$ \\
\hline$V_{t}$ & $\gamma_{0}$ & $\phi$ & -1 & - & - & + & + & $\mathrm{s}$ & $\mathrm{S}$ & $\mathrm{y}$ & $\mathrm{e}$ \\
$V_{s}$ & $\vec{\gamma}$ & $\vec{A}$ & +1 & + & + & + & - & $\mathrm{s}$ & $\mathrm{A}$ & $\mathrm{n}$ & $(\mathrm{e}, \mathrm{o}, \mathrm{e})$ \\
$B$ & $\gamma_{0} \vec{\gamma}$ & $\vec{E}$ & +1 & + & + & - & + & $\mathrm{s}$ & $\mathrm{A}$ & $\mathrm{n}$ & $(\mathrm{e}, \mathrm{o}, \mathrm{e})$ \\
$B$ & $\gamma_{14} \gamma_{0} \vec{\gamma}$ & $\vec{B}$ & -1 & - & - & - & - & $\mathrm{s}$ & $\mathrm{S}$ & $\mathrm{y}$ & $(\mathrm{o}, \mathrm{e}, \mathrm{o})$ \\
$A_{t}$ & $\gamma_{14} \gamma_{0}$ & $\phi_{m}$ & -1 & - & + & + & - & $\mathrm{a}$ & $\mathrm{A}$ & $\mathrm{y}$ & $\mathrm{o}$ \\
$A_{s}$ & $\gamma_{14} \vec{\gamma}$ & $\vec{A}_{m}$ & +1 & + & - & + & + & $\mathrm{a}$ & $\mathrm{S}$ & $\mathrm{n}$ & $(\mathrm{o}, \mathrm{e}, \mathrm{o})$ \\
$P$ & $\gamma_{14}$ & & -1 & - & + & - & + & $\mathrm{a}$ & $\mathrm{A}$ & $\mathrm{y}$ & $\mathrm{o}$ \\
$S$ & $\gamma_{15}=\mathbf{1}$ & & +1 & + & - & - & - & $\mathrm{a}$ & $\mathrm{S}$ & $\mathrm{n}$ & $\mathrm{e}$ \\
\hline \hline
\end{tabular}


the author prefers a unique and short name. A matrix $\mathbf{F}_{a}$ that holds

$$
\mathbf{F}_{a}^{T}=-\gamma_{0} \mathbf{F}_{a} \gamma_{0}
$$

is called an antisymplex. $\gamma_{0}$ itself is a symplex as it is antisymmetric and squares to the negative unit matrix. By definition the basic matrices $\gamma_{1}, \ldots, \gamma_{3}$ are also symplices.

The most important property of symplices is the superposition principle. According to this principle the sum of two symplices is a symplex:

$$
\begin{aligned}
\left(\mathbf{F}_{1}+\mathbf{F}_{2}\right)^{T} & =\mathbf{F}_{1}^{T}+\mathbf{F}_{2}^{T}=\gamma_{0} \mathbf{F}_{1} \gamma_{0}+\gamma_{0} \mathbf{F}_{2} \gamma_{0} \\
& =\gamma_{0}\left(\mathbf{F}_{1}+\mathbf{F}_{2}\right) \gamma_{0} .
\end{aligned}
$$

The superposition principle includes scalability: A symplex multiplied by a scalar is still a symplex. Given that the product of two symplices $\mathbf{F}_{1}$ and $\mathbf{F}_{2}$ is also a symplex, then one finds

$$
\begin{gathered}
\gamma_{0}\left(\mathbf{F}_{1} \mathbf{F}_{2}\right) \gamma_{0}=\left(\mathbf{F}_{1} \mathbf{F}_{2}\right)^{T}=\mathbf{F}_{2}^{T} \mathbf{F}_{1}^{T}=\gamma_{0} \mathbf{F}_{2} \gamma_{0} \gamma_{0} \mathbf{F}_{1} \gamma_{0} \\
=-\gamma_{0} \mathbf{F}_{2} \mathbf{F}_{1} \gamma_{0} \\
\mathbf{F}_{1} \mathbf{F}_{2}=-\mathbf{F}_{2} \mathbf{F}_{1}, \quad \mathbf{F}_{1} \mathbf{F}_{2}+\mathbf{F}_{2} \mathbf{F}_{1}=0 .
\end{gathered}
$$

The product of two symplices is a symplex, if (and only if) the symplices anticommute. Since the four basic matrices $\gamma_{0} \ldots \gamma_{3}$ anticommute with each other, the six possible bivectors $\gamma_{\nu} \gamma_{\mu}$ are symplices.

Since a symmetric $n \times n$ matrix $\mathbf{A}$ is described by $\nu$ parameters with

$$
\nu=n(n+1) / 2,
$$

the force matrix $\mathbf{F}=\gamma_{0} \mathbf{A}$ must contain the same number of independent components. In case of $n=4$ we expect $\nu=$ 10 force components. These are the four basic matrices, and the mentioned six bivectors $\gamma_{\nu} \gamma_{\mu}=\gamma_{4} \ldots \gamma_{9}$. Hence, a general force matrix in two dimensions has the form

$$
\mathbf{F}=\sum_{k=0}^{9} f_{k} \gamma_{k}
$$

\section{Symmetric products and projectors}

The symmetric product of a matrix $\mathbf{F}_{1}$, which is either a symplex or an antisymplex, and a symplex $\mathbf{F}_{2}$ is again a symplex:

$$
\begin{aligned}
\left(\mathbf{F}_{1} \mathbf{F}_{2} \mathbf{F}_{1}\right)^{T} & =\mathbf{F}_{1}^{T} \mathbf{F}_{2}^{T} \mathbf{F}_{1}^{T}=\left( \pm \gamma_{0} \mathbf{F}_{1} \gamma_{0}\right)\left(\gamma_{0} \mathbf{F}_{2} \gamma_{0}\right)\left( \pm \gamma_{0} \mathbf{F}_{1} \gamma_{0}\right) \\
& =\gamma_{0}\left(\mathbf{F}_{1} \mathbf{F}_{2} \mathbf{F}_{1}\right) \gamma_{0} .
\end{aligned}
$$

Since all $\gamma$ matrices are either symplices or antisymplices, any expression of the form

$$
\sum_{k=0}^{15} a_{k} \gamma_{k} \mathbf{F} \gamma_{k}
$$

with arbitrary coefficients $a_{k}$ is a symplex, if $\mathbf{F}$ is a symplex. Table I shows the result of $\gamma_{a} \gamma_{x} \gamma_{a}$ for $a=$ $[0,10,14]$. The result of the operation

$$
\mathbf{F}_{a}=\frac{\mathbf{F} \pm \gamma_{a} \mathbf{F} \gamma_{a}}{2}=\frac{1}{2} \sum_{k=0}^{9} f_{k}\left(\gamma_{k} \pm \gamma_{a} \gamma_{k} \gamma_{a}\right)
$$

is a projection. For $a=14$, for instance, one has

$$
\begin{aligned}
& \frac{1}{2} \sum_{k=0}^{9} f_{k}\left(\gamma_{k}+\gamma_{14} \gamma_{k} \gamma_{14}\right)=\sum_{k=0}^{3} f_{k} \gamma_{k} \\
& \frac{1}{2} \sum_{k=0}^{9} f_{k}\left(\gamma_{k}-\gamma_{14} \gamma_{k} \gamma_{14}\right)=\sum_{k=4}^{9} f_{k} \gamma_{k},
\end{aligned}
$$

that is, $\gamma_{14}$ separates the "vector" components from the "bivector" components.

\section{The transfer matrix}

The solution of Eq. (18) can be written by a symplectic transfer matrix $\mathbf{M}\left(t, t_{0}\right)$ :

$$
\psi(t)=\mathbf{M}\left(t, t_{0}\right) \psi\left(t_{0}\right) .
$$

If the force matrix is constant in time, then

$$
\mathbf{M}\left(t, t_{0}\right)=\exp \left[\mathbf{F}\left(t-t_{0}\right)\right] .
$$

The time derivative of Eq. (28) is

$$
\dot{\psi}(t)=\dot{\mathbf{M}}\left(t, t_{0}\right) \psi\left(t_{0}\right)=\mathbf{F} \psi(t)=\mathbf{F} \mathbf{M}\left(t, t_{0}\right) \psi\left(t_{0}\right)
$$

so that the following differential equation holds for $\mathbf{M}$ :

$$
\dot{\mathbf{M}}=\mathbf{F M} \text {. }
$$

Comparison with Eq. (18) shows that the column vectors of the transfer matrix $\mathbf{M}$ are solutions of Eq. (18). If $n$ is the dimension of $\psi$, the complete transfer matrix can be obtained by integrating Eq. (18) $n$ times, using the $n$ Euclidean unit vectors as starting conditions $\psi\left(t_{0}\right)$.

If the transfer matrix is expressed by a time-dependent matrix $\boldsymbol{\Phi}$ according to

$$
\begin{aligned}
& \mathbf{M}=\exp \boldsymbol{\Phi}=\sum_{k=0}^{\infty} \frac{\boldsymbol{\Phi}^{k}}{k !} \\
& \dot{\mathbf{M}}=\dot{\boldsymbol{\Phi}}+\frac{\dot{\boldsymbol{\Phi}} \boldsymbol{\Phi}+\boldsymbol{\Phi} \dot{\boldsymbol{\Phi}}}{2}+\frac{\dot{\boldsymbol{\Phi}} \boldsymbol{\Phi}^{2}+\boldsymbol{\Phi} \dot{\boldsymbol{\Phi}} \boldsymbol{\Phi}+\boldsymbol{\Phi}^{2} \dot{\boldsymbol{\Phi}}}{6}+\cdots,
\end{aligned}
$$

then if (and only if) $\boldsymbol{\Phi}$ and $\dot{\boldsymbol{\Phi}}$ commute, i.e., if

$$
\Phi \dot{\Phi}=\dot{\Phi} \Phi,
$$

Eq. (32) can be written as

$$
\dot{\mathbf{M}}=\dot{\boldsymbol{\Phi}}\left(\mathbf{1}+\boldsymbol{\Phi}+\frac{\boldsymbol{\Phi}^{2}}{2}+\frac{\boldsymbol{\Phi}^{3}}{6}+\cdots\right)=\dot{\boldsymbol{\Phi}} \exp \boldsymbol{\Phi}=\dot{\boldsymbol{\Phi}} \mathbf{M},
$$

so that in this case, one finds

$$
\mathbf{F}=\dot{\boldsymbol{\Phi}} \quad \boldsymbol{\Phi}\left(t, t_{0}\right)=\int_{t_{0}}^{t} \mathbf{F}(t) d t=\overline{\mathbf{F}} \tau,
$$

with $\tau=t-t_{0}$ so that the transfer matrix can be written as 


$$
\mathbf{M}(t)=\exp (\overline{\mathbf{F}} \tau)
$$

If $\boldsymbol{\Phi}$ and $\dot{\boldsymbol{\Phi}}$ would anticommute, i.e., if

$$
\boldsymbol{\Phi} \dot{\boldsymbol{\Phi}}+\dot{\boldsymbol{\Phi} \Phi}=0,
$$

then the square-and any even power of - $\boldsymbol{\Phi}$ would be constant and therefore one would find

$$
\dot{\mathbf{M}}=\sum_{k=0}^{\infty} \frac{\boldsymbol{\Phi}^{2 k} \dot{\boldsymbol{\Phi}}}{(2 k+1) !}=\sinh (\boldsymbol{\Phi}) \boldsymbol{\Phi}^{-1} \dot{\boldsymbol{\Phi}} .
$$

We define the matrices $\mathbf{M}_{s}$ and $\mathbf{M}_{c}$ according to

$$
\begin{aligned}
\mathbf{M} & =\mathbf{M}_{s}+\mathbf{M}_{c} \\
\mathbf{M}_{s} & \equiv \frac{\exp (\boldsymbol{\Phi})-\exp (-\boldsymbol{\Phi})}{2}=\frac{\mathbf{M}-\mathbf{M}^{-1}}{2}=\sinh (\boldsymbol{\Phi}) \\
\mathbf{M}_{c} & \equiv \frac{\exp (\boldsymbol{\Phi})+\exp (-\boldsymbol{\Phi})}{2}=\frac{\mathbf{M}+\mathbf{M}^{-1}}{2}=\cosh (\boldsymbol{\Phi})
\end{aligned}
$$

so that Eq. (37) would yield

$$
\dot{\mathbf{M}}_{c}=0 \quad \dot{\mathbf{M}}_{s}=\mathbf{M}_{s} \boldsymbol{\Phi}^{-1} \dot{\boldsymbol{\Phi}} .
$$

In Sec. II H it will become clear that Eq. (37) has to be rejected in the case of stable focused systems.

Beam transfer lines and circular accelerators are typically composed of guiding elements that provide a constant force matrix for a certain time (or better: length). Examples are dipole or quadrupole magnets, drifts and bends. In this case, it is possible to express the transfer matrix as a product of transfer matrices for the individual elements:

$$
\begin{aligned}
\mathbf{M}\left(t_{n}, t_{0}\right) & =\mathbf{M}\left(t_{n}, t_{n-1}\right) \ldots \mathbf{M}\left(t_{k}, t_{k-1}\right) \ldots \mathbf{M}\left(t_{1}, t_{0}\right) \\
& =\exp \left(\mathbf{F}_{n} \tau_{n}\right) \ldots \exp \left(\mathbf{F}_{1} \tau_{1}\right) .
\end{aligned}
$$

The transfer matrix is symplectic, if $\boldsymbol{\Phi}$ is a symplex:

$$
\begin{aligned}
\mathbf{M} \gamma_{0} \mathbf{M}^{T} & =\left(\sum_{k=0}^{\infty} \frac{\boldsymbol{\Phi}^{k}}{k !}\right) \gamma_{0}\left(\sum_{k=0}^{\infty} \frac{\left(\boldsymbol{\Phi}^{T}\right)^{k}}{k !}\right) \\
& =\left(\sum_{k=0}^{\infty} \frac{\boldsymbol{\Phi}^{k}}{k !}\right) \gamma_{0}\left(\sum_{k=0}^{\infty}(-)^{k-1} \gamma_{0} \frac{\boldsymbol{\Phi}^{k}}{k !} \gamma_{0}\right) \\
& =\left(\sum_{k=0}^{\infty} \frac{\boldsymbol{\Phi}^{k}}{k !}\right)\left(\sum_{k=0}^{\infty} \frac{(-\boldsymbol{\Phi})^{k}}{k !} \gamma_{0}\right) \\
& =\exp (\boldsymbol{\Phi}) \exp (-\boldsymbol{\Phi}) \gamma_{0}=\gamma_{0} .
\end{aligned}
$$

The exponential of a symplex is symplectic.

\section{E. The definition of coupling}

Before starting an investigation on decoupling there should be a clear definition of coupling. A possible (and typical) definition refers to the structure of the force matrix. As we defined 2 degrees of freedom $q_{1}$ and $q_{2}$, the obvious form of decoupling is a block-diagonal force matrix:

$$
\mathbf{F}=\left(\begin{array}{cc}
\mathbf{A} & 0 \\
0 & \mathbf{B}
\end{array}\right)
$$

where $\mathbf{A}$ and $\mathbf{B}$ are $2 \times 2$ matrices.

There are less obvious forms of decoupling - consider a constant force matrix. The second derivative of the state vector $\psi$ is

$$
\ddot{\psi}=\mathbf{F} \dot{\psi}=\mathbf{F}^{2} \psi .
$$

If the square of a constant force matrix is (block) diagonal, then the system can be regarded as decoupled in second order. The solutions can be found separately for each degree of freedom. In this case the coupling fixes the relative phases between the different degrees of freedom-but it does not determine the functional form of the solution.

A force matrix of the Dirac form is an example:

$$
\begin{aligned}
\mathbf{F} & =E \gamma_{0}+p_{1} \gamma_{1}+p_{2} \gamma_{2}+p_{3} \gamma_{3} \\
\mathbf{F}^{2} & =-\left(E^{2}-p_{1}^{2}-p_{2}^{2}-p_{3}^{2}\right) \mathbf{1} .
\end{aligned}
$$

Even though the odd component $p_{2} \gamma_{2}$ is not block diagonal, the second-order differential equation is decoupled. Another interesting example is a constant force matrix $\mathbf{X}$ of the form

$$
\mathbf{X}=-\frac{\gamma_{0}-\gamma_{2}-\gamma_{6}-\gamma_{7}}{2} \quad \mathbf{X}^{2}=-\gamma_{11} \quad \mathbf{X}^{4}=\mathbf{1} .
$$

Here one finds that neither $\mathbf{X}$ nor $\mathbf{X}^{2}$ or $\mathbf{X}^{3}$ are block diagonal. Nevertheless the fourth time derivative of $\psi$ is "decoupled."

We refer to systems as decoupled in $\mathrm{n}$ th order, if the $n$th order EQOM have the form

$$
\left(\frac{d}{d \tau}\right)^{n} \psi=\mathbf{B} \psi
$$

where $\mathbf{B}$ is block diagonal. In case of time-dependent force matrices, the second derivative is

$$
\ddot{\psi}=\dot{\mathbf{F}} \psi+\mathbf{F} \dot{\psi}=\left(\dot{\mathbf{F}}+\mathbf{F}^{2}\right) \psi .
$$

Hence, we consider systems with time-dependent force matrices to be decoupled to second order, if the expression $\dot{\mathbf{F}}+\mathbf{F}^{2}$ is block diagonal.

\section{F. Symplectic symplices}

Table I lists all RDMs with their main properties. Each RDM is either a symplex or an antisymplex, symplectic or antisymplectic. Four RDMs are both-symplectic and symplex. If $\mathbf{F}$ is a symplectic symplex (SYSY), then the combination of Eqs. (42) and (19) yields

$$
\mathbf{F} \gamma_{0} \mathbf{F}^{T}=\mathbf{F} \gamma_{0} \gamma_{0} \mathbf{F} \gamma_{0}=-\mathbf{F}^{2} \gamma_{0}=\gamma_{0} \Rightarrow \mathbf{F}^{2}=-\mathbf{1} .
$$

A symplex is symplectic, if (and only if) its square equals the negative unit matrix. But note that symplectic matrices are in principle not scalable, i.e., without unit. Symplices 
are scalable and may therefore have arbitrary units. If the symplex $\mathbf{F}$ appears in the form of Eq. (18), then it has the natural unit of a frequency or wave number if the dot is interpreted as time or path length derivative, respectively. In practical problems of accelerator physics, it will always be possible to find a typical length or time interval that can be used to redefine the dot derivative in such a way that the force matrix is unitless. In consequence, this means that force matrices which fulfill $\mathbf{F}^{2}=-\Omega^{2} \mathbf{1}$ with a constant $\Omega$ are equivalent to SYSYs:

$$
\begin{aligned}
\dot{\psi}=\frac{d}{d t} \psi & =\mathbf{F} \psi \\
\Rightarrow \quad \frac{d}{d \tau} \psi & =\frac{\mathbf{F}}{\Omega} \psi \\
d \tau & =\Omega d t .
\end{aligned}
$$

In this respect the Dirac operator [Eq. (45)] is a SYSY.

From Eqs. (29) and (49) it is quickly derived that the transfer matrix of a constant SYSY $\mathbf{F}$ is given by

$$
\mathbf{M}=\mathbf{1} \cos t+\mathbf{F} \sin t \text {. }
$$

Equation (51) is known in the Courant-Synder theory of one-dimensional ion beam optics. The unitless parameter $t$ is called phase advance. If $t$ represents the phase advance for one turn, then it is called tune. In the one-dimensional theory, the matrix $\mathbf{F}$ has the form

$$
\mathbf{F}=\left(\begin{array}{cc}
\alpha & \beta \\
-\gamma & -\alpha
\end{array}\right)
$$

where $\alpha, \beta$, and $\gamma$ are the so-called Twiss parameters [20]. According to the concept of Sec. IIE, a constant SYSY is decoupled in second order and Eq. (51) shows that this definition is meaningful. Besides this, a SYSY also has equal eigenfrequencies - as will be shown in Sec. II Gand in consequence there is a common phase advance for both degrees of freedom.

Let $\mathbf{U}$ be a time-dependent symplectic transformation, then we obtain for the transformed "spinor" $\tilde{\psi}$ :

$$
\tilde{\psi}=\mathbf{U} \psi \quad \dot{\tilde{\psi}}=\dot{\mathbf{U}} \psi+\mathbf{U} \dot{\psi} .
$$

The time derivative of Eq. (18) is

$$
\ddot{\psi}=\dot{\mathbf{F}} \psi+\mathbf{F} \dot{\psi} .
$$

The formal difference between Eqs. (53) and (54) is that $\mathbf{U}$ is symplectic and $\mathbf{F}$ is a symplex. If $\mathbf{U}$ is also a symplex (or if $\mathbf{F}$ is symplectic), then these equations through light on the structural equivalence between a (time) derivative and the multiplication with a SYSY. If the dynamics of a system is described by a SYSY, then the (time) derivative is itself a symplectic transformation. One could say that SYSYs are operators, which are equivalent to derivatives.

\section{G. Eigenvalues and eigenvectors}

Eigenvalues and eigenvectors play an important role, if the system has some kind of self-feedback. Circular accelerators like storage rings are a simple example for a system with self-feedback. Another example are systems with a constant force matrix, so-called "constant focusing channels." If $\lambda$ is the diagonal matrix containing the eigenvalues of $\mathbf{F}$ and $\mathbf{E}$ is the matrix of columnwise eigenvectors, then

$$
\mathbf{F} \mathbf{E}=\mathbf{E} \lambda .
$$

If $\mathbf{E}$ can be reversed, then

$$
\mathbf{F}=\mathbf{E} \lambda \mathbf{E}^{-1} .
$$

In the simplest case of a constant force matrix, one finds that

$$
\begin{aligned}
\mathbf{M}(\tau, 0) & =\exp \left(\mathbf{E} \lambda \mathbf{E}^{-1} \tau\right)=\sum_{k=0}^{\infty} \frac{\left(\mathbf{E} \lambda \mathbf{E}^{-1} \tau\right)^{k}}{k !} \\
& =\sum_{k=0}^{\infty} \mathbf{E} \frac{\lambda^{k} \tau^{k}}{k !} \mathbf{E}^{-1}=\mathbf{E} \exp (\lambda \tau) \mathbf{E}^{-1} \\
& =\mathbf{E} \Lambda(\tau) \mathbf{E}^{-1}
\end{aligned}
$$

where

$$
\Lambda(\tau)=\exp (\lambda \tau)
$$

is the diagonal matrix of the eigenvalues of $\mathbf{M}$.

The trace of the product of an antisymmetric and a symmetric matrix is zero. Hence, $\mathbf{F}$ has zero trace. As similarity transformations preserve the trace and the eigenvalues, we find $\operatorname{Tr}(\lambda)=\operatorname{Tr}(\mathbf{F})=0$ and, hence, the sum of all eigenvalues is zero. The eigenvalues are either real or (two) pairs of complex conjugate values. A symplectic transformation is said to be strongly stable, if all eigenvalues of $\mathbf{M}$ are distinct and lie on the unit circle in the complex plane [18]. This means that for stable (oscillatory) solutions the eigenvalues of $\mathbf{F}$ are two conjugate pairs of imaginary values:

$$
\begin{aligned}
\lambda & =\operatorname{Diag}\left(i \omega_{1},-i \omega_{1}, i \omega_{2},-i \omega_{2}\right) \\
& =-i \frac{\omega_{1}+\omega_{2}}{2} \gamma_{3}-i \frac{\omega_{1}-\omega_{2}}{2} \gamma_{4} .
\end{aligned}
$$

Equation (56) yields

$$
\begin{aligned}
\mathbf{F}^{2} & =\mathbf{E} \lambda^{2} \mathbf{E}^{-1}=-\mathbf{E}\left(\frac{\omega_{1}^{2}+\omega_{2}^{2}}{2} \mathbf{1}+\frac{\omega_{1}^{2}-\omega_{2}^{2}}{2} \gamma_{12}\right) \mathbf{E}^{-1} \\
& =-\frac{\omega_{1}^{2}+\omega_{2}^{2}}{2} \mathbf{1}-\frac{\omega_{1}^{2}-\omega_{2}^{2}}{2} \mathbf{E} \gamma_{12} \mathbf{E}^{-1}
\end{aligned}
$$

This shows that $\mathbf{F}$ is (isomorphic to) a SYSY, if $\omega_{1}^{2}=\omega_{2}^{2}$. SYSYs are degenerate. The absolute values of all eigenfrequencies of a SYSY are equal.

\section{H. The form of the transfer matrix}

Equation (36) allows specific functional forms $f(\tau)$ for the matrix elements-depending on the dimensionality and the properties of the force matrix. The force matrix of a 
strongly stable system has eigenvalues that are grouped in two pairs of imaginary values - the eigenfrequencies. Ring accelerators always have this property-but not in each section. Ion beam transport systems are usually composed of sections with separate elements like dipole, quadrupole, or sextupole magnets, drifts, solenoids, and so on. These elements are characterized by their individual transfer matrices and are not necessarily "stable." Only the product of the transfer matrices of all elements in a ring accelerator has to be stable. Equation (36) is actually computed as a series:

$$
\mathbf{M}=\exp (\overline{\mathbf{F}} \tau)=\sum_{k=0}^{\infty} \frac{(\overline{\mathbf{F}} \tau)^{k}}{k !} .
$$

In the case of pure RDMs, i.e. $\overline{\mathbf{F}}=\gamma_{k}$ with $k \in[0, \ldots, 9]$, the functional form of the elements of the corresponding transfer matrices are exponentials of $\tau$-if we include (hyperbolic) sine and cosine forms. In the case of arbitrary symplices, other forms are possible: A square matrix $\mathbf{F}$ is called nilpotent, if $\mathbf{F}^{q}=0$ for some positive integer $q>1$. A simple example is the "force" matrix of a drift, which is given by [see Eq. (133) in Sec. III G below]

$$
\mathbf{F}=\frac{\gamma_{0}+\gamma_{6}}{2} \quad \mathbf{M}=\sum_{k=0}^{\infty} \frac{(\overline{\mathbf{F}} \tau)^{k}}{k !}=\mathbf{1}+\overline{\mathbf{F}} \tau .
$$

Since the maximal (nonzero) power of a nilpotent $n \times n$ matrix $\mathbf{F}$ is $n-1$, polynomials up to third order as well as products of polynomials and exponentials are also possible solutions of Eq. (61).

In the case of a constant force or in the case of a transfer matrix for a complete turn in a stable circular accelerator, the diagonalized transfer matrix can be computed from Eqs. (58) and (59). We introduce the abbreviations

$$
\begin{aligned}
& \Sigma_{c}=\frac{\cos \left(\omega_{1} \tau\right)+\cos \left(\omega_{2} \tau\right)}{2}=\cos (\bar{\omega} \tau) \cos (\Delta \omega \tau) \\
& \Sigma_{s}=\frac{\sin \left(\omega_{1} \tau\right)+\sin \left(\omega_{2} \tau\right)}{2}=\sin (\bar{\omega} \tau) \cos (\Delta \omega \tau) \\
& \Delta_{s}=\frac{\sin \left(\omega_{1} \tau\right)-\sin \left(\omega_{2} \tau\right)}{2}=\cos (\bar{\omega} \tau) \sin (\Delta \omega \tau) \\
& \Delta_{c}=\frac{\cos \left(\omega_{1} \tau\right)-\cos \left(\omega_{2} \tau\right)}{2}=-\sin (\bar{\omega} \tau) \sin (\Delta \omega \tau),
\end{aligned}
$$

where

$$
\bar{\omega}=\frac{\omega_{1}+\omega_{2}}{2} \quad \Delta \omega=\frac{\omega_{1}-\omega_{2}}{2}
$$

so that the diagonal matrix $\Lambda$ [Eqs. (57) and (58)] can be written as

$$
\Lambda=\Sigma_{c} \mathbf{1}-i \Sigma_{s} \gamma_{3}-i \Delta_{s} \gamma_{4}-\Delta_{c} \gamma_{12},
$$

and the one-turn-transfer matrix of strongly stable systems is according to Eq. (57) given by

$$
\mathbf{M}=\Sigma_{c} \mathbf{1}-i \Sigma_{s} \mathbf{E} \gamma_{3} \mathbf{E}^{-1}-i \Delta_{s} \mathbf{E} \gamma_{4} \mathbf{E}^{-1}-\Delta_{c} \mathbf{E} \gamma_{12} \mathbf{E}^{-1} \text {. }
$$

Equation (66) is the generalization of the Twiss matrix for two-dimensional systems [21]. It shows that transfer matrices usually have-in contrast to symplices-a scalar component as well as components which are antisymplices. The second and the third term of Eq. (66) have the same form as a force matrix (though different eigenvalues). The last term vanishes in the case of a degenerate system with equal eigenfrequencies. In this case the one-turn transfer matrix has the form of Eq. (51).

In order to split the transfer matrix into the components according to Eq. (66), we make use of the method of projections, modified for this purpose, as follows: The inverse of a symplectic (transfer) matrix $\mathbf{M}$ has the same eigenvectors as $\mathbf{M}$ :

$$
\mathbf{M}^{-1}=\mathbf{E} \Lambda^{-1}(\tau) \mathbf{E}^{-1}=\mathbf{E} \Lambda(-\tau) \mathbf{E}^{-1}=\mathbf{E} \exp (-\lambda \tau) \mathbf{E}^{-1},
$$

so that using Eq. (17) yields

$$
\frac{1}{2}\left(\mathbf{M} \pm \mathbf{M}^{-1}\right)=\mathbf{E} \frac{\Lambda(\tau) \pm \Lambda(-\tau)}{2} \mathbf{E}^{-1}=\frac{1}{2}\left(\mathbf{M} \mp \gamma_{0} \mathbf{M}^{T} \gamma_{0}\right) .
$$

If we recall Eq. (39), then we can determine the RDM coefficients of the transfer matrix and the matrices $\mathbf{M}_{s}$ and $\mathbf{M}_{c}$ according to Eqs. (10) and (11). Let $m_{k}$ be the resulting coefficients, then insertion into Eq. (68) yields

$$
\begin{aligned}
\mathbf{M}_{s} & =\frac{1}{2}\left(\mathbf{M}+\gamma_{0} \mathbf{M}^{T} \gamma_{0}\right)=\sum_{k=0}^{9} m_{k} \gamma_{k}=\mathbf{E} \frac{\Lambda(\tau)-\Lambda(-\tau)}{2} \mathbf{E}^{-1} \\
& =-i \Sigma_{s} \mathbf{E} \gamma_{3} \mathbf{E}^{-1}-i \Delta_{s} \mathbf{E} \gamma_{4} \mathbf{E}^{-1} \\
\mathbf{M}_{c} & =\frac{1}{2}\left(\mathbf{M}-\gamma_{0} \mathbf{M}^{T} \gamma_{0}\right)=\sum_{k=10}^{15} m_{k} \gamma_{k}=\mathbf{E} \frac{\Lambda(\tau)+\Lambda(-\tau)}{2} \mathbf{E}^{-1} \\
& =\Sigma_{c} \mathbf{1}-\Delta_{c} \mathbf{E} \gamma_{12} \mathbf{E}^{-1}
\end{aligned}
$$

A decoupled force matrix logically implies a decoupled transfer matrix. A comparison of Eqs. (56), (59), and (69) shows that the force matrix and $\mathbf{M}_{s}$ differ only in the eigenvalues. Hence, all the information that is required to compute the decoupling transformation can be obtained either from the force matrix or from $\mathbf{M}_{s}$. $\mathbf{M}_{c}$ can be ignored in the context of decoupling. Even more than that: The matrix of eigenvectors $\mathbf{E}$ of the matrix $\mathbf{M}_{s}$ diagonalizes $\mathbf{M}_{c}$, too. We come back to this in Secs. IV D and IV F, after the construction of the matrix of eigenvectors $\mathbf{E}$.

From Eq. (69), one quickly derives that $\mathbf{M}_{s}$ is a symplex while $\mathbf{M}_{c}$ is an antisymplex. As $\mathbf{M}_{s}$ and $\mathbf{M}_{c}$ share the same eigenvectors and since all diagonal matrices, i.e. $\gamma_{3}, \gamma_{4}, \gamma_{12}$ and the unit matrix commute, also $\mathbf{M}_{s}$ and $\mathbf{M}_{c}$ commute:

$$
\mathbf{M}_{s} \mathbf{M}_{c}-\mathbf{M}_{c} \mathbf{M}_{s}=0 .
$$

From Eqs. (15) and (70), one derives

$$
\mathbf{M}_{c}^{2}-\mathbf{M}_{s}^{2}=\mathbf{1}
$$


which includes that

$$
1=\Sigma_{c}^{2}+\Delta_{c}^{2}+\Sigma_{s}^{2}+\Delta_{s}^{2} \quad 0=\Sigma_{c} \Delta_{c}+\Sigma_{s} \Delta_{s},
$$

in agreement with Eq. (63).

\section{Second moments and the envelope equations}

Besides the position of a beam relative to the design orbit, the most important properties of an ion beam are collected in the matrix of second moments. We assume in the following that the beam is centered, i.e., that the first moments are all identically zero. If the state vectors $\psi_{i}(\tau)$ with $i=1, \ldots, n$ represent a family of $n$ ions, where $\tau$ is the path length along the reference orbit, then the matrix of second moments $\sigma$ is given by

$$
\sigma=\frac{1}{n} \sum_{i=1}^{n} \psi_{i} \psi_{i}^{T}=\left\langle\psi \psi^{T}\right\rangle .
$$

Another possible parametrization is given by a density function $\rho(\psi, \tau)=\rho\left(q_{1}, p_{1}, q_{2}, p_{2}, \tau\right)$ which should be normalized such that

$$
\int \ldots \int \rho\left(q_{1}, p_{1}, q_{2}, p_{2}, \tau\right) d q_{1} d p_{1} d q_{2} d p_{2}=1 .
$$

In this case, one writes

$$
\sigma=\int \ldots \int \rho \psi \psi^{T} d q_{1} d p_{1} d q_{2} d p_{2} .
$$

But independent of the specific practical method of computation, we assume that the matrix of second moments $\sigma=\left\langle\psi \psi^{T}\right\rangle$ is well defined and has a nonvanishing determinant. From Eq. (18) one derives

$$
\begin{aligned}
\dot{\sigma} & =\left\langle\dot{\psi} \psi^{T}\right\rangle+\left\langle\psi \dot{\psi}^{T}\right\rangle=\left\langle\mathbf{F} \psi \psi^{T}\right\rangle+\left\langle\psi \psi^{T} \mathbf{F}^{T}\right\rangle \\
& =\mathbf{F} \sigma+\sigma \mathbf{F}^{T}=\mathbf{F} \sigma+\sigma \gamma_{0} \mathbf{F} \gamma_{0} .
\end{aligned}
$$

We define the $\mathbf{S}$ matrix using $\bar{\psi} \equiv \psi^{T} \gamma_{0}$ by

$$
\mathbf{S} \equiv \sigma \gamma_{0}=\left\langle\psi \psi^{T} \gamma_{0}\right\rangle=\langle\psi \bar{\psi}\rangle,
$$

and obtain from Eq. (76) by multiplication from the right with $\gamma_{0}$ :

$$
\dot{\mathbf{S}}=\mathbf{F S}-\mathbf{S F} .
$$

In ion beam physics, Eq. (76) is called an envelope equation, as the second moments define the envelope of an ion beam. $\mathbf{S}$ is a symplex-as any symmetric matrix multiplied by $\gamma_{0}$ :

$$
\mathbf{S}^{T}=\gamma_{0}^{T} \sigma^{T}=-\gamma_{0} \sigma=\gamma_{0} \sigma \gamma_{0}^{2}=\gamma_{0} \mathbf{S} \gamma_{0} .
$$

Assuming a constant force matrix F, Eq. (78) tells us that the second moments are constant, if $\mathbf{S}$ and $\mathbf{F}$ commute. Using the eigenvector decomposition, Eq. (56) of $\mathbf{F}$ gives

$$
\begin{aligned}
& 0=\mathbf{F S}-\mathbf{S F}=\mathbf{E} \lambda \mathbf{E}^{-1} \mathbf{S}-\mathbf{S E} \lambda \mathbf{E}^{-1} \\
& 0=\lambda \mathbf{E}^{-1} \mathbf{S E}-\mathbf{E}^{-1} \mathbf{S E} \lambda \quad 0=\lambda \tilde{\mathbf{S}}-\tilde{\mathbf{S}} \lambda .
\end{aligned}
$$

Since $\lambda$ is diagonal and $\tilde{\mathbf{S}}$ commutes with $\lambda$, also

$$
\tilde{\mathbf{S}}=\mathbf{E}^{-1} \mathbf{S E}=\mathbf{D}
$$

must be diagonal (see Table IV), so that the matrix $\mathbf{S}$ has the form

$$
\mathbf{S}=\mathbf{E D E}^{-1} .
$$

The force matrix and the $\mathbf{S}$ matrix share the same eigenvectors-but will in general have different eigenvalues.

\section{J. Matching}

Matching is a concept of circular accelerators, i.e., systems with self-feedback, where eigenvalues and vectors are well defined. A beam is matched, if the phase space occupied by the ions in the beam fits to the "acceptance" of the machine. The practical consequence of mismatching is an oscillation or "pumping" of the phase-space distribution which typically leads to an increase of the beam emittance by filamentation [20].

Wolski formulated the state of matching for the general case of periodic motion [11]. In this case, the restriction is no more that $\mathbf{F}$ has to be constant, but that $\mathbf{F}$ must be periodic: $\mathbf{F}(\tau+C)=\mathbf{F}(\tau)$ for a given period $C$ and any $\tau$. Given the transfer matrix over one turn (or period) is $\mathbf{M}$, then the beam is matched, if

$$
\sigma=\mathbf{M} \sigma \mathbf{M}^{T}
$$

Using Eq. (17), one quickly finds in analogy to Eq. (80):

$$
\begin{aligned}
& \sigma=-\mathbf{M} \sigma \gamma_{0} \mathbf{M}^{-1} \gamma_{0} \quad \sigma \gamma_{0}=\mathbf{M} \sigma \gamma_{0} \mathbf{M}^{-1} \\
& \mathbf{S}=\mathbf{M S M}^{-1} \quad \mathbf{S M}=\mathbf{M S} \quad 0=\mathbf{M S}-\mathbf{S M} .
\end{aligned}
$$

Following the same arguments as for Eq. (80), one finds that the matrix of second moments is matched, if the transfer matrix over one period and the $\mathbf{S}$ matrix share the same system of eigenvectors. The general form of the matrix $\mathbf{S}$ is again given by Eq. (82) where $\mathbf{D}$ has a form analogue to Eq. (59) and is given by [11]

$$
\mathbf{D}=\operatorname{Diag}\left(i \varepsilon_{1},-i \varepsilon_{1}, i \varepsilon_{2},-i \varepsilon_{2}\right) .
$$

\section{K. Expectation values and scalar product}

The "expectation values" $\left\langle\bar{\psi} \gamma_{x} \psi\right\rangle$ are related to the $\mathbf{S}$ matrix according to 


$$
\begin{aligned}
& \bar{\psi} \gamma_{x} \psi= \sum_{i j k} \psi^{k} \gamma_{0}^{k i} \gamma_{x}^{i j} \psi^{j} \\
&= \frac{1}{2} \sum_{i j k}\left(\psi^{k} \gamma_{0}^{k i} \gamma_{x}^{i j} \psi^{j}+\psi^{j} \gamma_{0}^{j k} \gamma_{x}^{k i} \psi^{i}\right) \\
&= \frac{1}{2} \sum_{i j k}\left(\gamma_{x}^{i j} \psi^{j} \psi^{k} \gamma_{0}^{k i}+\psi^{i} \psi^{j} \gamma_{0}^{j k} \gamma_{x}^{k i}\right) \\
&= \frac{1}{2} \operatorname{Tr}\left(\sum_{j k}\left(\gamma_{x}^{i j} \psi^{j} \psi^{k} \gamma_{0}^{k l}+\psi^{i} \psi^{j} \gamma_{0}^{j k} \gamma_{x}^{k l}\right)\right) \\
&= \frac{1}{2} \operatorname{Tr}\left(\gamma_{x} \psi \bar{\psi}+\psi \bar{\psi} \gamma_{x}\right)=\frac{1}{2} \operatorname{Tr}\left(\gamma_{x} \mathbf{S}+\mathbf{S} \gamma_{x}\right), \\
& \mathbf{D}=\operatorname{Diag}\left(i \varepsilon_{1},-i \varepsilon_{1}, i \varepsilon_{2},-i \varepsilon_{2}\right),
\end{aligned}
$$

where the $\varepsilon_{i}$ are the emittances of the two degrees of freedom. The RDM coefficients of the $\mathbf{S}$ matrix are-apart from the sign - the expectation values of the RDMs [see Eq. (11)].

\section{POISSON BRACKETS OF SECOND MOMENTS AND THE ELECTROMECHANICAL EQUIVALENCE}

The total time derivative of a function $f(p, q, t)$ is given by the Poisson brackets with the Hamiltonian function [22]:

$$
\begin{aligned}
\frac{d f\left(q_{i}, p_{i}, t\right)}{d t} & =\frac{\partial f}{\partial t}+\sum_{i}\left\{\frac{\partial f}{\partial q_{i}} \frac{\partial H}{\partial p_{i}}-\frac{\partial f}{\partial p_{i}} \frac{\partial H}{\partial q_{i}}\right\} \\
& =\left(\begin{array}{c}
\frac{\partial f}{\partial q_{1}} \\
\frac{\partial f}{\partial p_{1}} \\
\frac{\partial f}{\partial q_{2}} \\
\frac{\partial f}{\partial p_{2}}
\end{array}\right) \gamma_{0}\left(\begin{array}{c}
\frac{\partial H}{\partial q_{1}} \\
\frac{\partial H}{\partial p_{1}} \\
\frac{\partial H}{\partial q_{2}} \\
\frac{\partial H}{\partial p_{2}}
\end{array}\right)=\frac{\partial f}{\partial t}+\nabla_{q, p} f(p, q, t) \dot{\psi} \\
& =\frac{\partial f}{\partial t}+\nabla_{q, p} f(p, q, t) \mathbf{F} \psi
\end{aligned}
$$

We define the functions $f_{k}$ by the "expectation values" according to

$$
f_{k}(p, q)=\frac{1}{2} \bar{\psi} \gamma_{k} \psi
$$

$f_{k}(p, q)$ do not explicitly depend on time. Evidently the $f_{k}$ vanish for all nonsymmetric matrices $\gamma_{0} \gamma_{k}$ so that there should be exactly $n(n+1) / 2=10$ nonvanishing functions $f_{k}$. The gradient $\nabla_{p, q}=\nabla_{\psi}$ yields

$$
\partial_{\psi_{i}} \psi_{j}=\delta_{i j}
$$

so that

$$
\begin{aligned}
\nabla_{p, q} f_{k} & =\frac{1}{2}\left(\gamma_{0} \gamma_{k} \psi+\psi^{T} \gamma_{0} \gamma_{k}\right)=\frac{1}{2} \psi^{T}\left[\gamma_{0} \gamma_{k}+\left(\gamma_{0} \gamma_{k}\right)^{T}\right] \\
& =\frac{1}{2} \psi^{T}\left(\gamma_{0} \gamma_{k}+\gamma_{k}^{T} \gamma_{0}^{T}\right)=\frac{1}{2} \psi^{T}\left(\gamma_{0} \gamma_{k}-\gamma_{k}^{T} \gamma_{0}\right) \\
& =\frac{1}{2} \psi^{T}\left(\gamma_{0} \gamma_{k}+\gamma_{0} \gamma_{0} \gamma_{k}^{T} \gamma_{0}\right)=\frac{1}{2} \bar{\psi}\left(\gamma_{k}+\gamma_{0} \gamma_{k}^{T} \gamma_{0}\right)
\end{aligned}
$$

Since all $\gamma$ matrices are either symplices or antisymplices, it is obvious that

$$
\nabla_{p, q} f_{k}= \begin{cases}\bar{\psi} \gamma_{k} & \text { for } k \in[0 \ldots 9] \\ 0 & \text { for } k \in[10 \ldots 15],\end{cases}
$$

i.e., only symplices can have a nonvanishing expectation value. For all symplices $\gamma_{k}$ with $k \in[0, \ldots, 9]$ the Poisson brackets result:

$$
\frac{d}{d \tau}\left(\frac{\bar{\psi} \gamma_{k} \psi}{2}\right)=\bar{\psi} \gamma_{k} \mathbf{F} \psi
$$

On the other hand, we find from Eq. (18)

$$
\begin{aligned}
\frac{d}{d \tau}\left(\bar{\psi} \gamma_{k} \psi\right) & =\dot{\psi}^{T} \gamma_{0} \gamma_{k} \psi+\psi^{T} \gamma_{0} \gamma_{k} \dot{\psi} \\
& =\psi^{T} \mathbf{F}^{T} \gamma_{0} \gamma_{k} \psi+\psi^{T} \gamma_{0} \gamma_{k} \mathbf{F} \psi \\
& =\psi^{T} \gamma_{0} \mathbf{F} \gamma_{0} \gamma_{0} \gamma_{k} \psi+\psi^{T} \gamma_{0} \gamma_{k} \mathbf{F} \psi \\
& =-\bar{\psi} \mathbf{F} \gamma_{k} \psi+\bar{\psi} \gamma_{k} \mathbf{F} \psi \\
& =\bar{\psi}\left(\gamma_{k} \mathbf{F}-\mathbf{F} \gamma_{k}\right) \psi
\end{aligned}
$$

Combining Eqs. (92) and (93) yields

$$
\frac{d}{d \tau}\left(\bar{\psi} \gamma_{k} \psi\right)=\bar{\psi} 2 \gamma_{k} \mathbf{F} \psi=\bar{\psi}\left(\gamma_{k} \mathbf{F}-\mathbf{F} \gamma_{k}\right) \psi,
$$

so that

$0=\bar{\psi} 2 \gamma_{k} \mathbf{F} \psi-\bar{\psi}\left(\gamma_{k} \mathbf{F}-\mathbf{F} \gamma_{k}\right) \psi=\bar{\psi}\left(\gamma_{k} \mathbf{F}+\mathbf{F} \gamma_{k}\right) \psi$.

According to Eq. (24), the force matrix $\mathbf{F}$ can be written as

$$
\mathbf{F}=\sum_{l=0}^{9} F_{l} \gamma_{l}
$$

so that Eq. (93) can be written as

$$
\dot{f}_{k}=\frac{d}{d \tau}\left(\frac{\bar{\psi} \gamma_{k} \psi}{2}\right)=\sum_{l=0}^{9} \bar{\psi} \frac{\gamma_{k} \gamma_{l}-\gamma_{l} \gamma_{k}}{2} \psi F_{l}=\sum_{l=0}^{9} \mathbf{G}_{k l} F_{l} .
$$

The matrix $\mathbf{G}_{k l}$ is composed of the expectation values $f_{i}$ and the indices of $f_{i}$ are given by the upper left $10 \times 10$ part of the commutator table (Table IV). Note that all commutators of symplices are either zero or again symplices.

Equation (97) can be reordered such that it has the form of a linear transformation of a ten-dimensional vector $f_{i}$ :

$$
\dot{f}_{i}=\sum_{j=0}^{9} \mathbf{B}_{i j} f_{j} .
$$

The matrix $\mathbf{G}_{k l}$ is antisymmetric. The reordering results in a quite similar matrix $\mathbf{B}_{i j}$. Now we introduce a normalization that assures a positive sign for positive definite second moments. For instance, $f_{0}$ is according to the definition given by 


$$
f_{0}=\bar{\psi} \gamma_{0} \psi=\psi^{T} \gamma_{0}^{2} \psi=-\psi^{T} \psi,
$$

which is negative even though the corresponding second moment is positive. The normalization is done by multiplication with $\gamma_{k}^{2}= \pm 1$, which equals -1 for $k \in$ $\{0,7,8,9\}$. This can be expressed by the multiplication with a quadratic diagonal matrix $\tilde{g}$ that is an extended version of the "metric tensor" $g_{\mu \nu}$ :

$$
\tilde{g}=\operatorname{Diag}(-1,1,1,1,1,1,1,-1,-1,-1) \quad \tilde{g}^{2}=\mathbf{1} .
$$

The transformed EQOM are

$$
\dot{f}=\mathbf{B} f \quad \tilde{g} \dot{f}=(\tilde{g} \mathbf{B} \tilde{g})(\tilde{g} f) \quad \dot{\tilde{f}}=\tilde{\mathbf{B}} \tilde{f},
$$

where the matrix $\tilde{\mathbf{B}}$ is explicitly given by

$$
\tilde{\mathbf{B}}=\left(\begin{array}{cccccccccc} 
& F_{4} & F_{5} & F_{6} & -F_{1} & -F_{2} & -F_{3} & & & \\
F_{4} & & F_{9} & -F_{8} & -F_{0} & & & & F_{3} & -F_{2} \\
F_{5} & -F_{9} & & F_{7} & & -F_{0} & & -F_{3} & & F_{1} \\
F_{6} & F_{8} & -F_{7} & & & & -F_{0} & F_{2} & -F_{1} & \\
-F_{1} & F_{0} & & & & F_{9} & -F_{8} & & F_{6} & -F_{5} \\
-F_{2} & & F_{0} & & -F_{9} & & F_{7} & -F_{6} & & F_{4} \\
-F_{3} & & & F_{0} & F_{8} & -F_{7} & & F_{5} & -F_{4} & \\
& & -F_{3} & F_{2} & & -F_{6} & F_{5} & & F_{9} & -F_{8} \\
& F_{3} & & -F_{1} & F_{6} & & -F_{4} & -F_{9} & & F_{7}
\end{array}\right) .
$$

Equation (101) is another way to express the envelope equations [Eqs. (76) and (78)]. The explicit relation between the second moments $\sigma_{i j}$ and the expectation values is given in Appendix E.

\section{A. Symplectic electrodynamics}

The upper left $4 \times 4$ block of the matrix $\tilde{\mathbf{B}}$ equals the electromagnetic field tensor, if we replace the vector $\left(F_{4}, F_{5}, F_{6}\right)^{T}$ by the electric field $\vec{E}$ and $\left(F_{7}, F_{8}, F_{9}\right)^{T}$ by the magnetic field $\vec{B}$. In this section we investigate the equivalence of two-dimensional symplectic flow and relativistic electrodynamics. In the next section we show that rotations and Lorentz boosts in Minkowski space are formally identical to a subset of symplectic transformations in two-dimensional coupled linear optics.

If one writes the 4-potential $\boldsymbol{\Phi}$ (4-current $\mathbf{J}$, 4momentum $\mathbf{P}$ ) as a 4-vector using the RDMs $\gamma_{0} \ldots \gamma_{3}$ according to

$$
\boldsymbol{\Phi}=\phi \gamma_{0}+A_{x} \gamma_{1}+A_{y} \gamma_{2}+A_{z} \gamma_{3}=\phi \gamma_{0}+\vec{\gamma} \vec{A},
$$

the 4-derivative $\mathbf{D}$ as

$$
\mathbf{D}=\partial_{t} \gamma_{0}-\partial_{x} \gamma_{1}-\partial_{y} \gamma_{2}-\partial_{z} \gamma_{3},
$$

and the electromagnetic fields $\vec{E}$ and $\vec{B}$ as

$$
\mathbf{F}=E_{x} \gamma_{4}+E_{y} \gamma_{5}+E_{z} \gamma_{6}+B_{x} \gamma_{7}+B_{y} \gamma_{8}+B_{z} \gamma_{9},
$$

then the Maxwell equations (MWEQs) can be written (remarkably compact) as

$$
\mathbf{F}=-\mathbf{D \Phi} \quad \mathbf{D F}=4 \pi \mathbf{J},
$$

with the usual choice of units.

The Lorentz force can also be expressed by RDMs. If the 4-momentum is defined by

$$
\mathbf{P}=\mathcal{E} \gamma_{0}+p_{x} \gamma_{1}+p_{y} \gamma_{2}+p_{z} \gamma_{3}=\mathcal{E} \gamma_{0}+\vec{p} \vec{\gamma},
$$

where $\mathcal{E}$ is the energy and $\vec{p}$ the momentum, then the Lorentz force equations can be written as

$$
\frac{d \mathbf{P}}{d \tau}=\dot{\mathbf{P}}=\frac{q}{2 m}(\mathbf{F P}-\mathbf{P F}),
$$

where $\tau$ is the proper time. In the lab frame time $d t=\frac{d \tau}{\gamma}$, Eq. (108) yields (setting $c=1$ )

$$
\begin{array}{rlrl}
\frac{d \mathcal{E}}{d \tau} & =\frac{q}{m} \vec{p} \vec{E} & & \frac{d \vec{p}}{d \tau}=\frac{q}{m}(\mathcal{E} \vec{E}+\vec{p} \times \vec{B}) \\
\gamma \frac{d \mathcal{E}}{d t} & =q \gamma \vec{v} \vec{E} & \gamma \frac{d \vec{p}}{d t}=\frac{q}{m}(m \gamma \vec{E}+m \gamma \vec{v} \times \vec{B}) \\
\frac{d E}{d t} & =q \vec{v} \vec{E} & & \frac{d \vec{p}}{d t}=q(\vec{E}+\vec{v} \times \vec{B}),
\end{array}
$$

which are exactly the Lorentz force equations.

We use the formal identity of Eqs. (78) and (108) to introduce the electromechanical equivalence (EMEQ) of two-dimensional symplectic motion and the motion of charged particles in electromagnetic fields as described by the Lorentz force equations. The statement of the EMEQ is that the transformation properties of the elements of the $\mathbf{S}$ matrix with respect to symplectic transformations are formally identical to the transformation properties of momentum $\mathbf{P}$ and electromagnetic field $\mathbf{F}_{e d}$ in Minkowski space. The analogy allows one to obtain an overview over the components of the force matrix $\mathbf{F}$ and to establish a meaningful nomenclature for the RDM coefficients: The ten elements of the force matrix are associated with the energy and momentum of - and with electric and magnetic field components "seen by" —a relativistic charged particle: 


$$
\begin{aligned}
\mathbf{F}= & \mathcal{E} \gamma_{0}+p_{x} \gamma_{1}+p_{y} \gamma_{2}+p_{z} \gamma_{3}+E_{x} \gamma_{4}+E_{y} \gamma_{5} \\
& +E_{z} \gamma_{6}+B_{x} \gamma_{7}+B_{y} \gamma_{8}+B_{z} \gamma_{9} .
\end{aligned}
$$

If the force matrix is split into the electrodynamical $\mathbf{F}_{e d}$ and the mechanical components $\mathbf{F}_{m}$, the matrices are explicitly given by

$$
\begin{aligned}
\mathbf{F}= & \mathbf{F}_{e d}+\mathbf{F}_{m} \\
= & \left(\begin{array}{cccc}
-E_{x} & E_{z}+B_{y} & E_{y}-B_{z} & B_{x} \\
E_{z}-B_{y} & E_{x} & -B_{x} & -E_{y}-B_{z} \\
E_{y}+B_{z} & B_{x} & E_{x} & E_{z}-B_{y} \\
-B_{x} & -E_{y}+B_{z} & E_{z}+B_{y} & -E_{x}
\end{array}\right) \\
& +\left(\begin{array}{cccc}
-p_{z} & \mathcal{E}-p_{x} & 0 & p_{y} \\
-\mathcal{E}-p_{x} & p_{z} & -p_{y} & 0 \\
0 & p_{y} & -p_{z} & \mathcal{E}+p_{x} \\
p_{y} & 0 & -\mathcal{E}+p_{x} & p_{z}
\end{array}\right) .
\end{aligned}
$$

Note that there are ten force terms in symplectic motion and only six electromagnetic components. Correspondingly, there are ten symplectic transformations in two-dimensional symplectic flow, but only six transformations known in Minkowski space-rotations about and boosts along three axis. One also finds that Eqs. (101) and (102) correspond to the Lorentz force equations only if $F 0, F 1, F 2$, and $F 3$ are zero. In this case $\tilde{\mathbf{B}}$ is block diagonal - the first block being the upper left $4 \times 4$ matrix and the second block the lower right $6 \times 6$ matrix. Coupled linear motion in two dimensions does not include such restrictions and is in this sense a richer theory.

Nevertheless, let us emphasize that the theory of symplectic motion in two dimensions does not allow scalar source terms, i.e., nonvanishing coefficients of $\gamma_{15}$ in the force matrix. If the MWEQs are derived from twodimensional symplectic motion, then the Lorentz gauge is included. To make this clearer, we look at the first part of Eq. (106), which includes a scalar expression $\partial_{t} \phi+\vec{\nabla} \vec{A}$ :

$$
\begin{aligned}
\mathbf{F} & =-\mathbf{D} \boldsymbol{\Phi}=-\left(\partial_{t} \gamma_{0}-\vec{\gamma} \vec{\nabla}\right)\left(\phi \gamma_{0}+\vec{\gamma} \vec{A}\right) \\
& =\partial_{t} \phi+\vec{\nabla} \vec{A}-\left(\partial_{t} \vec{A}+\vec{\nabla} \phi\right) \gamma_{0} \vec{\gamma}+(\vec{\nabla} \times \vec{A}) \gamma_{14} \gamma_{0} \vec{\gamma} \\
& =\left(\partial_{t} \phi+\vec{\nabla} \vec{A}\right) \mathbf{1}+\vec{E} \gamma_{0} \vec{\gamma}+\vec{B} \gamma_{14} \gamma_{0} \vec{\gamma},
\end{aligned}
$$

where $\vec{\gamma}=\left(\gamma_{1}, \gamma_{2}, \gamma_{3}\right)^{T}$. Since the force matrix may be composed exclusively of symplices, the scalar term has to vanish, i.e.:

$$
\partial_{t} \phi+\vec{\nabla} \vec{A}=0
$$

which is the so-called "Lorentz gauge." In Sec. III F, it will be shown that the so-called duality rotation also has properties which are incompatible to the presented theory of two-dimensional symplectic motion.

In the next section we give a survey of symplectic transformations using the EMEQ according to Eq. (110).
This concept allows one to obtain a very transparent and systematic treatment of two-dimensional coupled linear optics. Of course there is some danger of puzzling the formally introduced electromagnetic components of the EMEQ with the fields of the accelerator due to the similarity of notation.

\section{B. A Survey of symplectic transformations}

Symplectic transformations have been introduced by Eqs. (18) and (28). It was shown that the exponentials of symplices are symplectic and that there are ten symplices (in two dimensions) corresponding to ten free parameters in symplectic matrices. This indicates a one-to-one relation between the symplices and the corresponding symplectic matrix. In the following we will classify the transformations that are based on single $\gamma$ matrices. Six of these transformations are known as rotations and Lorentz boosts in three-dimensional space, based on the six bivectors $\gamma_{4}, \ldots, \gamma_{9}$, i.e., the "electric and magnetic field components." We call the four remaining transformations phase rotation (induced by $\gamma_{0}$, i.e., by "energy") and phase boosts induced by the spatial basis vectors $\gamma_{1}, \ldots, \gamma_{3}$ ("momentum"), respectively. All transformations are controlled by a continuous parameter $a$ such that the inverse transformation is given by the negative of this parameter and a vanishing parameter $a=0$ yields the unit matrix:

$$
\mathbf{R}(0)=\mathbf{1} \quad \mathbf{R}(a)^{-1}=\mathbf{R}(-a) .
$$

The parameter $a$ has a specific physical meaning in the EMEQ. In case of rotations it is the angle, in case of Lorentz boosts it is the inverse hyperbolic tangent of $\beta=v / c$.

Before discussing the action of the individual $\gamma$ symplices, we look again at the Hamiltonian in order to show exactly why the transformation matrix should be symplectic:

$$
H(p, q)=\psi^{T} \mathbf{A} \psi=-\psi^{T} \gamma_{0} \mathbf{F} \psi,
$$

and insert the equation for a symplectic transformation of the forces $\mathbf{F}^{\prime}=\mathbf{R} \mathbf{F} \mathbf{R}^{-1}$, then we find (using $\bar{\psi} \equiv \psi^{T} \gamma_{0}$ )

$$
\begin{aligned}
H(p, q) & =-\bar{\psi} \mathbf{R}^{-1} \mathbf{R} \mathbf{F} \mathbf{R}^{-1} \mathbf{R} \psi \\
& =-\left(\bar{\psi} \mathbf{R}^{-1}\right)\left(\mathbf{R} \mathbf{F} \mathbf{R}^{-1}\right)(\mathbf{R} \psi) \\
& =\left(\bar{\psi} \mathbf{R}^{-1}\right) \gamma_{0} \gamma_{0}\left(\mathbf{R} \mathbf{F} \mathbf{R}^{-1}\right)(\mathbf{R} \psi) \\
& =-\left(\bar{\psi} \mathbf{R}^{-1} \gamma_{0}\right) \mathbf{A}^{\prime} \psi^{\prime}=\psi^{\prime T} \mathbf{A}^{\prime} \psi^{\prime},
\end{aligned}
$$

that the Hamiltonian is invariant under Lorentz transformations and under the assumption that the spinors transform according to

$$
\begin{aligned}
\mathbf{F}^{\prime} & =\mathbf{R} \mathbf{F} \mathbf{R}^{-1} \quad \psi^{\prime}=\mathbf{R} \psi \\
\psi^{\prime T} & =-\psi^{T} \gamma_{0} \mathbf{R}^{-1} \gamma_{0}=\psi^{T} \mathbf{R}^{T} \\
& \Rightarrow-\gamma_{0} \mathbf{R}^{-1} \gamma_{0}=\mathbf{R}^{T} \Rightarrow \gamma_{0}=\mathbf{R}^{T} \gamma_{0} \mathbf{R}
\end{aligned}
$$

or, in other words: If a transformation matrix $\mathbf{R}$ is symplectic, then the form (and the value) of the Hamiltonian and of 
the EQOM is conserved. Note that if the force matrix is transformed according to Eq. (117), then we find for the transfer matrix:

$$
\begin{aligned}
\mathbf{M}^{\prime} & =\exp \left(\mathbf{F}^{\prime} s\right)=\sum_{k=0}^{\infty} \frac{\left(\mathbf{F}^{\prime} s\right)^{k}}{k !}=\sum_{k=0}^{\infty} \frac{\left(\mathbf{R} \mathbf{F} \mathbf{R}^{-1} s\right)^{k}}{k !} \\
& =\mathbf{R}\left(\sum_{k=0}^{\infty} \frac{(\mathbf{F} s)^{k}}{k !}\right) \mathbf{R}^{-1}=\mathbf{R} \mathbf{M} \mathbf{R}^{-1}
\end{aligned}
$$

Symplectic transformation matrices have the form

$$
\mathbf{R}=\exp (\mathbf{F} \boldsymbol{\varepsilon}) \quad \mathbf{R}^{-1}=\exp (-\mathbf{F} \boldsymbol{\varepsilon})
$$

where the matrix $\mathbf{F}$ is a symplex. The effect of a basic symplex $\gamma_{b}$ is given by

$$
\begin{aligned}
\mathbf{R}_{b} & =\exp \left(\gamma_{b} \varepsilon\right) \\
\mathbf{R}_{b} & =\sum_{k=0}^{\infty} \frac{\left(\gamma_{b} \varepsilon\right)^{2 k}}{(2 k) !}+\sum_{k=0}^{\infty} \frac{\left(\gamma_{b} \varepsilon\right)^{2 k+1}}{(2 k+1) !} \\
& =\sum_{k=0}^{\infty}\left(\gamma_{b}^{2}\right)^{k} \frac{\varepsilon^{2 k}}{(2 k) !}+\gamma_{b} \sum_{k=0}^{\infty}\left(\gamma_{b}^{2}\right)^{k} \frac{\varepsilon^{2 k+1}}{(2 k+1) !} \\
& = \begin{cases}\mathbf{1} \cos (\varepsilon)+\gamma_{b} \sin (\varepsilon) & \text { for } \gamma_{b}^{2}=-1 \\
\mathbf{1} \cosh (\varepsilon)+\gamma_{b} \sinh (\varepsilon) & \text { for } \gamma_{b}^{2}=1\end{cases} \\
\mathbf{R}_{b}^{-1} & =\exp \left(-\gamma_{b} \varepsilon\right) \\
& = \begin{cases}\mathbf{1} \cos (\varepsilon)-\gamma_{b} \sin (\varepsilon) & \text { for } \gamma_{b}^{2}=-1 \\
\mathbf{1} \cosh (\varepsilon)-\gamma_{b} \sinh (\varepsilon) & \text { for } \gamma_{b}^{2}=1 .\end{cases}
\end{aligned}
$$

Transformations with $\gamma_{b}^{2}=-1$ are orthogonal transformations, i.e., rotations, while those with $\gamma_{b}^{2}=1$ are boosts. According to Table I, $\gamma_{b}$ with $b \in[0,7,8,9]$ produce rotations and $\gamma_{b}$ with $b \in[1, \ldots, 6]$ boosts. Hence, the transformed matrices are

$$
\begin{aligned}
\gamma_{a}^{\prime} & =\mathbf{R}^{-1} \gamma_{a} \mathbf{R}=\mathbf{R}^{-1} \gamma_{a} \mathbf{R}=\left(c-s \gamma_{b}\right) \gamma_{a}\left(c+s \gamma_{b}\right) \\
& =c^{2} \gamma_{a}-\gamma_{b} \gamma_{a} \gamma_{b} s^{2}+c s\left(\gamma_{a} \gamma_{b}-\gamma_{b} \gamma_{a}\right)
\end{aligned}
$$

where $c$ and $s$ are the (hyperbolic) sine and cosine functions according to Eq. (120). The last term on the right vanishes if $\gamma_{b}$ and $\gamma_{a}$ commute. In this case the matrix $\gamma_{a}$ remains unchanged:

$$
\begin{aligned}
\gamma_{a}^{\prime} & =\gamma_{a}\left(c^{2}-\gamma_{b}^{2} s^{2}\right) \\
& =\gamma_{a} \begin{cases}\cos ^{2}(\varepsilon)+\sin ^{2}(\varepsilon)=1 & \text { for } \gamma_{b}^{2}=-1 \\
\cosh ^{2}(\varepsilon)-\sinh ^{2}(\varepsilon)=1 & \text { for } \gamma_{b}^{2}=1 .\end{cases}
\end{aligned}
$$

If the RDMs $\gamma_{b}$ and $\gamma_{a}$ anticommute, one finds

$$
\begin{aligned}
\gamma_{a}^{\prime} & =\gamma_{a}\left(c^{2}+\gamma_{b}^{2} s^{2}\right)+2 c s \gamma_{a} \gamma_{b} \\
& = \begin{cases}\gamma_{a} \cos (2 \varepsilon)+\gamma_{a} \gamma_{b} \sin (2 \varepsilon) & \text { for } \gamma_{b}^{2}=-1 \\
\gamma_{a} \cosh (2 \varepsilon)+\gamma_{a} \gamma_{b} \sinh (2 \varepsilon) & \text { for } \gamma_{b}^{2}=1 .\end{cases}
\end{aligned}
$$

The elementary symplectic transformations (in two dimensions) are summarized in Table II. The "frequency doubling" is an indication that the transformed elements are according to their transformation properties second moments. This is usually taken into account by using a transformation with the "half-angle."

Note that, even though we describe here the transforma-

\begin{tabular}{|c|c|c|c|c|c|c|c|c|c|c|}
\hline & $\gamma_{0}$ & $\gamma_{1}$ & $\gamma_{2}$ & $\gamma_{3}$ & $\gamma_{4}$ & $\gamma_{5}$ & $\gamma_{6}$ & $\gamma_{7}$ & $\gamma_{8}$ & $\gamma_{9}$ \\
\hline$c=$ & $\cos \varepsilon$ & $\cosh \varepsilon$ & $\cosh \varepsilon$ & $\cosh \varepsilon$ & $\cosh \varepsilon$ & $\cosh \varepsilon$ & $\cosh \varepsilon$ & $\cos \varepsilon$ & $\cos \varepsilon$ & $\cos \varepsilon$ \\
\hline$s=$ & $\sin \varepsilon$ & $\sinh \varepsilon$ & $\sinh \varepsilon$ & $\sinh \varepsilon$ & $\sinh \varepsilon$ & $\sinh \varepsilon$ & $\sinh \varepsilon$ & $\sin \varepsilon$ & $\sin \varepsilon$ & $\sin \varepsilon$ \\
\hline$\gamma_{0}^{\prime}=c \gamma_{0} \ldots$ & & $+s \gamma_{4}$ & $+s \gamma_{5}$ & $+s \gamma_{6}$ & $-s \gamma_{1}$ & $-s \gamma_{2}$ & $-s \gamma_{3}$ & & & \\
\hline$\gamma_{1}^{\prime}=c \gamma_{1} \ldots$ & $-s \gamma_{4}$ & & $+s \gamma_{9}$ & $-s \gamma_{8}$ & $-s \gamma_{0}$ & & & & $-s \gamma_{3}$ & $+s \gamma_{2}$ \\
\hline$\gamma_{2}^{\prime}=c \gamma_{2} \ldots$ & $-s \gamma_{5}$ & $-s \gamma_{9}$ & & $+s \gamma_{7}$ & & $-s \gamma_{0}$ & & $+s \gamma_{3}$ & & $-s \gamma_{1}$ \\
\hline$\gamma_{3}^{\prime}=c \gamma_{3} \ldots$ & $-s \gamma_{6}$ & $+s \gamma_{8}$ & $-s \gamma_{7}$ & & & & $-s \gamma_{0}$ & $-s \gamma_{2}$ & $+s \gamma_{1}$ & \\
\hline$\gamma_{4}^{\prime}=c \gamma_{4} \ldots$ & $+s \gamma_{1}$ & $+s \gamma_{0}$ & & & & $+s \gamma_{9}$ & $-s \gamma_{8}$ & & $-s \gamma_{6}$ & $\gamma_{5}$ \\
\hline$\gamma_{5}^{\prime}=c \gamma_{5} \ldots$ & $+s \gamma_{2}$ & & $+s \gamma_{0}$ & & $-s \gamma_{9}$ & & $+s \gamma_{7}$ & $+s \gamma_{6}$ & & $-s \gamma_{4}$ \\
\hline$\gamma_{6}^{\prime}=c \gamma_{6} \ldots$ & $+s \gamma_{3}$ & & & $+s \gamma_{0}$ & $+s \gamma_{8}$ & $-s \gamma_{7}$ & & $-s \gamma_{5}$ & $+s \gamma_{4}$ & \\
\hline$\gamma_{7}^{\prime}=c \gamma_{7} \ldots$ & & & $-s \gamma_{3}$ & $+s \gamma_{2}$ & & $-s \gamma_{6}$ & $+s \gamma_{5}$ & & $-s \gamma_{9}$ & $+s \gamma_{8}$ \\
\hline$\gamma_{8}^{\prime}=c \gamma_{8} \ldots$ & & $+s \gamma_{3}$ & & $-s \gamma_{1}$ & $+s \gamma_{6}$ & & $-s \gamma_{4}$ & $+s \gamma_{9}$ & & $-s \gamma_{7}$ \\
\hline$\gamma_{9}^{\prime}=c \gamma_{9} \ldots$ & & $-s \gamma_{2}$ & $+s \gamma_{1}$ & & $-s \gamma_{5}$ & $+s \gamma_{4}$ & & $-s \gamma_{8}$ & $+s \gamma_{7}$ & \\
\hline
\end{tabular}
tion of the matrices instead of the RDM coefficients, this is equivalent to the transformation of the RDM coefficients assuming that the RDMs have the same form in all coordinate systems. This is only a matter of notation.

\section{Rotations and Lorentz boosts}

Lorentz boosts are all transformations induced by $\gamma_{b}$ with $b \in[4, \ldots, 6]$, i.e., by the electric field terms, while rotations are induced by $\gamma_{b}$ with $b \in[7, \ldots, 9]$, i.e., by magnetic field terms. Note that both transformations are induced by bivectors and not by the basic matrices.

TABLE II. Table of symplectic transformations in two dimensions. $a$ indicates the rows and $b$ the column: $\gamma_{a}^{\prime}=$ $\exp \left(-\gamma_{b} \varepsilon / 2\right) \gamma_{a} \exp \left(-\gamma_{b} \varepsilon / 2\right)$. If $\gamma_{a}$ and $\gamma_{b}$ anticommute, then the result is $\gamma_{a}^{\prime}=c \gamma_{a}+s \gamma_{a} \gamma_{b}$, where $c$ and $s$ are the sine and cosine function of $\varepsilon$, if $\gamma_{b}^{2}=-1$, and hyperbolic sine and cosine function if $\gamma_{b}^{2}=1$, respectively. If $\gamma_{a}$ and $\gamma_{b}$ commute, then $\gamma_{a}^{\prime}=\gamma_{a}$. The table follows the commutator table (Table IV). 
Both—rotations and Lorentz boosts—are well known [23] and the matrix coefficients transform exactly as the physical quantities associated by the EMEQ. Hence, we do not need to repeat the formulas here as all relevant properties are summarized in Table II.

Nevertheless it is instructive to look at the rotation matrices with respect to the transformation of the spinor $\psi$. The matrix of the spatial rotation about the $z$ axis, induced by $\gamma_{9}$, is explicitly given by

$$
\exp \left(\gamma_{9} \frac{\varepsilon}{2}\right)=\left(\begin{array}{cccc}
\cos \frac{\varepsilon}{2} & 0 & -\sin \frac{\varepsilon}{2} & 0 \\
0 & \cos \frac{\varepsilon}{2} & 0 & -\sin \frac{\varepsilon}{2} \\
\sin \frac{\varepsilon}{2} & 0 & \cos \frac{\varepsilon}{2} & 0 \\
0 & \sin \frac{\varepsilon}{2} & 0 & \cos \frac{\varepsilon}{2}
\end{array}\right)
$$

Obviously $\gamma_{9}$ induces a rotation in the plane of $q_{1}$ and $q_{2}$. The canonical momenta are rotated accordingly. Note that the rotation angle in the associated space is $\varepsilon$, the corresponding angle in the plane of the canonical variables is half of that: $\varepsilon / 2$.

The rotation about the $x$ axis $\left(\gamma_{7}\right)$ mixes the canonical coordinates and momenta $q_{1}$ and $p_{2}\left(p_{1}\right.$ and $\left.q_{2}\right)$ :

$$
\exp \left(\gamma_{7} \frac{\varepsilon}{2}\right)=\left(\begin{array}{cccc}
\cos \frac{\varepsilon}{2} & 0 & 0 & \sin \frac{\varepsilon}{2} \\
0 & \cos \frac{\varepsilon}{2} & -\sin \frac{\varepsilon}{2} & 0 \\
0 & \sin \frac{\varepsilon}{2} & \cos \frac{\varepsilon}{2} & 0 \\
-\sin \frac{\varepsilon}{2} & 0 & 0 & \cos \frac{\varepsilon}{2}
\end{array}\right)
$$

A phase rotation is a rotation in phase space, for instance a rotation in the plane of $q_{1}$ and $p_{2}$ as described by Eq. (125). Another example is given by the rotation about the $y$ axis as induced by $\gamma_{8}-\mathrm{a}$ combined phase rotation in opposite directions:

$$
\exp \left(\gamma_{8} \frac{\varepsilon}{2}\right)=\left(\begin{array}{cccc}
\cos \frac{\varepsilon}{2} & \sin \frac{\varepsilon}{2} & 0 & 0 \\
-\sin \frac{\varepsilon}{2} & \cos \frac{\varepsilon}{2} & 0 & 0 \\
0 & 0 & \cos \frac{\varepsilon}{2} & -\sin \frac{\varepsilon}{2} \\
0 & 0 & \sin \frac{\varepsilon}{2} & \cos \frac{\varepsilon}{2}
\end{array}\right)
$$

In combination with the phase rotation matrix $\gamma_{0}$, this gives

$$
\begin{aligned}
\exp \left(\left(\gamma_{0}+\gamma_{8}\right) \frac{\varepsilon}{2}\right) & =\left(\begin{array}{cccc}
\cos \varepsilon & \sin \varepsilon & 0 & 0 \\
-\sin \varepsilon & \cos \varepsilon & 0 & 0 \\
0 & 0 & 1 & 0 \\
0 & 0 & 0 & 1
\end{array}\right) \\
\exp \left(\left(\gamma_{0}-\gamma_{8}\right) \frac{\varepsilon}{2}\right) & =\left(\begin{array}{cccc}
1 & 0 & 0 & 0 \\
0 & 1 & 0 & 0 \\
0 & 0 & \cos \varepsilon & \sin \varepsilon \\
0 & 0 & -\sin \varepsilon & \cos \varepsilon
\end{array}\right),
\end{aligned}
$$

so that both degrees of freedom are here decoupled. Note that in this case, the rotation angle $\varepsilon$ is not divided by two.

We call the remaining symplectic transformations phase rotation induced by $\gamma_{0}$ and phase boosts induced by $\gamma_{1}, \gamma_{2}$, and $\gamma_{3}$. This is not quite correct, since we saw that also $\gamma_{8}$ induces a phase rotation. Strictly speaking, there is only one "real" spatial rotation possible in two dimensionsand this is induced by $\gamma_{9}$. All other "rotations" are rotations in phase space and are therefore phase rotations. Nevertheless, the rotations induced by $\gamma_{7}$ and $\gamma_{8}$ are spatial rotations in the context of the EMEQ.

\section{Phase rotation}

The symplectic transformation driven by $\gamma_{0}$ is a "rotation" between spacelike components and electric field components, represented by $\gamma_{0} \vec{\gamma} \cdot \gamma_{0}$ and the magnetic components $\gamma_{7}, \gamma_{8}, \gamma_{9}$ are unchanged. Expressing this using EMEQ, one may write

$E^{\prime}=E \quad \vec{p}^{\prime}=\cos \varepsilon \vec{p}-\sin \varepsilon \vec{E} \quad \vec{E}^{\prime}=\cos \varepsilon \vec{E}+\sin \varepsilon \vec{p} \quad \vec{B}^{\prime}=\vec{B}$.

The rotation matrix is given by

$$
\exp \left(\gamma_{0} \frac{\varepsilon}{2}\right)=\left(\begin{array}{cccc}
\cos \frac{\varepsilon}{2} & \sin \frac{\varepsilon}{2} & 0 & 0 \\
-\sin \frac{\varepsilon}{2} & \cos \frac{\varepsilon}{2} & 0 & 0 \\
0 & 0 & \cos \frac{\varepsilon}{2} & \sin \frac{\varepsilon}{2} \\
0 & 0 & -\sin \frac{\varepsilon}{2} & \cos \frac{\varepsilon}{2}
\end{array}\right)
$$

As listed in Table III, the matrices $\gamma_{0}, \gamma_{4}, \gamma_{5}, \gamma_{6}$ form an alternative basis to $\gamma_{0}, \gamma_{1}, \gamma_{2}, \gamma_{3}$. The phase rotation produced by $\gamma_{0}$ changes the "mixing" angle of the standard basis and this first alternative basis.

\section{E. Phase boost}

The transformations induced by $\gamma_{1}, \gamma_{2}$, and $\gamma_{3}$ are called "phase boost." These boosts are the exact analogue of the Lorentz boosts, but the transformation properties of $\vec{E}$ and $\vec{p}$ are exchanged, as can be seen from Table II. While Lorentz boosts are known to have the invariants $\vec{E} \vec{B}$ and $\vec{E}^{2}-\vec{B}^{2}$, a phase boost has the invariants $\vec{p} \vec{B}$ and $\vec{p}^{2}-\vec{B}^{2}$. Hence, the phase boosts transform mass and electromagnetic energy into each other as the Lorentz boosts are transforming mass and kinetic energy. We will come back to this in Secs. IV.

\section{F. The duality rotation}

The duality rotation can be expressed by the matrix $\gamma_{14}$, but it is not a symplectic transformation and has some surprising and puzzling features. Since $\gamma_{14}$ commutes with all electric and magnetic field components (see Table IV), the usual form of the transformation cannot transform the fields, but only the particle properties of energy and momentum. Using the nomenclature of Eq. (111), one finds 
TABLE III. List of the indices of equivalent canonical basis sets of $\gamma$ matrices and the corresponding reinterpretations of the matrices relative to the choice of the basis. Negative indices indicate the negative matrix with the index taken as absolute value. $\left(V_{0}, \vec{V}\right)$ are the elements corresponding to the so-called "axial vector," "PS" is the pseudoscalar.

\begin{tabular}{|c|c|c|c|c|c|c|c|c|c|c|c|c|c|c|c|}
\hline \multirow[b]{2}{*}{ (1) } & \multicolumn{4}{|c|}{$\begin{array}{c}\text { Basis }(\Phi, \vec{A}) \\
\text { 4-vector }\end{array}$} & \multicolumn{3}{|c|}{$\begin{array}{c}\vec{E} \\
\text { Electric field }\end{array}$} & \multicolumn{3}{|c|}{$\begin{array}{c}\vec{B} \\
\text { Magnetic field }\end{array}$} & \multicolumn{4}{|c|}{$\begin{array}{c}V_{0} \vec{V} \\
\text { Axial 4-vector }\end{array}$} & \multirow{2}{*}{$\begin{array}{l}\text { PS } \\
\text { PS } \\
14\end{array}$} \\
\hline & 0 & 1 & 2 & 3 & 4 & 5 & 6 & 7 & 8 & 9 & 10 & 11 & 12 & 13 & \\
\hline (2) & 0 & 4 & 5 & 6 & -1 & -2 & -3 & 7 & 8 & 9 & 14 & 11 & 12 & 13 & -10 \\
\hline (3) & 14 & 1 & 2 & 3 & 11 & 12 & 13 & 7 & 8 & 9 & 10 & -4 & -5 & -6 & -0 \\
\hline (4) & 14 & 11 & 12 & 13 & -1 & -2 & -3 & 7 & 8 & 9 & -0 & -4 & -5 & -6 & -10 \\
\hline (5) & 10 & 11 & 12 & 13 & 4 & 5 & 6 & 7 & 8 & 9 & -0 & -1 & -2 & -3 & 14 \\
\hline (6) & 10 & 4 & 5 & 6 & -11 & -12 & -13 & 7 & 8 & 9 & 14 & -1 & -2 & -3 & 0 \\
\hline (7) & 7 & 3 & 6 & 13 & 2 & 5 & 12 & 10 & 14 & -0 & 9 & -1 & -4 & -11 & 8 \\
\hline (8) & 7 & 2 & 5 & 12 & -3 & -6 & -13 & 10 & 14 & -0 & 8 & -1 & -4 & -11 & -9 \\
\hline (9) & 8 & 3 & 6 & 13 & -1 & -4 & -11 & 10 & 14 & -0 & 9 & -2 & -5 & -12 & -7 \\
\hline (10) & 8 & 1 & 4 & 11 & 3 & 6 & 13 & 10 & 14 & -0 & 7 & -2 & -5 & -12 & 9 \\
\hline (11) & 9 & 1 & 4 & 11 & -2 & -5 & -12 & 10 & 14 & -0 & 7 & -3 & -6 & -13 & -8 \\
\hline (12) & 9 & 2 & 5 & 12 & 1 & 4 & 11 & 10 & 14 & -0 & 8 & -3 & -6 & -13 & 7 \\
\hline
\end{tabular}

$$
\begin{aligned}
\mathbf{R} & =\exp \left(\gamma_{14} \varepsilon / 2\right) \quad \mathbf{R}^{-1}=\exp \left(-\gamma_{14} \varepsilon / 2\right) \\
\mathbf{F}_{e d} & =\mathbf{R} \mathbf{F}_{e d} \mathbf{R}^{-1}=\mathbf{R}^{-1} \mathbf{F}_{e d} \mathbf{R} \quad \mathbf{F}_{m}=\mathbf{R} \mathbf{F}_{m} \mathbf{R} \\
\mathbf{F}_{m}^{\prime} & =\mathbf{R} \mathbf{F}_{m} \mathbf{R}^{-1}=\cos \varepsilon \mathbf{F}_{m}+\sin \varepsilon \gamma_{14} \mathbf{F}_{m} \\
\mathbf{F}^{\prime}{ }_{e d} & =\mathbf{R} \mathbf{F}_{e d} \mathbf{R}=\cos \varepsilon \mathbf{F}_{e d}+\sin \varepsilon \gamma_{14} \mathbf{F}_{e d} .
\end{aligned}
$$

The last line of Eqs. (130) is usually referred to as duality rotation, explicitly given by

$$
\vec{E}^{\prime}=\cos \varepsilon \vec{E}-\sin \varepsilon \vec{B} \quad \vec{B}^{\prime}=\cos \varepsilon \vec{B}+\sin \varepsilon \vec{E}
$$

The duality rotation fails to fulfill all reasonable requirements in the context of the EMEQ. First, it is not symplectic.
Second, the transformation is not a similarity transformation and, finally, it has different forms for the mechanical and the electrodynamical components.

If the MWEQs are derivable from the EMEQ and symplectic flow, then there is no magnetic charge or current density, but the MWEQs are forced to have the "classical" form with

$$
\vec{\nabla} \vec{B}=0 \quad \vec{\nabla} \times \vec{E}+\partial_{t} \vec{B}=0 .
$$

\begin{tabular}{|c|c|c|c|c|c|c|c|c|c|c|c|c|c|c|c|c|}
\hline & 0 & 1 & 2 & 3 & 4 & 5 & 6 & 7 & 8 & 9 & 10 & 11 & 12 & 13 & 14 & 15 \\
\hline 0 & & 4 & 5 & 6 & -1 & -2 & -3 & & & & 14 & & & & -10 & \\
\hline 1 & -4 & & 9 & -8 & -0 & & & & -3 & 2 & & -14 & & & -11 & \\
\hline 2 & -5 & -9 & & 7 & & -0 & & 3 & & -1 & & & -14 & & -12 & \\
\hline 3 & -6 & 8 & -7 & & & & -0 & -2 & 1 & & & & & -14 & -13 & \\
\hline 4 & 1 & 0 & & & & 9 & -8 & & -6 & 5 & 11 & 10 & & & & \\
\hline 5 & 2 & & 0 & & -9 & & 7 & 6 & & -4 & 12 & & 10 & & & \\
\hline 6 & 3 & & & 0 & 8 & -7 & & -5 & 4 & & 13 & & & 10 & & \\
\hline 7 & & & -3 & 2 & & -6 & 5 & & -9 & 8 & & & -13 & 12 & & \\
\hline 8 & & 3 & & -1 & 6 & & -4 & 9 & & -7 & & 13 & & -11 & & \\
\hline 9 & & -2 & 1 & & -5 & 4 & & -8 & 7 & & & -12 & 11 & & & \\
\hline 10 & -14 & & & & -11 & -12 & -13 & & & & & 4 & 5 & 6 & 0 & \\
\hline 11 & & 14 & & & -10 & & & & -13 & 12 & -4 & & 9 & -8 & 1 & \\
\hline 12 & & & 14 & & & -10 & & 13 & & -11 & -5 & -9 & & 7 & 2 & \\
\hline 13 & & & & 14 & & & -10 & -12 & 11 & & -6 & 8 & -7 & & 3 & \\
\hline 14 & 10 & 11 & 12 & 13 & & & & & & & -0 & -1 & -2 & -3 & & \\
\hline 15 & & & & & & & & & & & & & & & & \\
\hline
\end{tabular}

To conclude, the duality rotation does not fit into in the concept of the EMEQ.

TABLE IV. Commutator table of the group of $\gamma$ matrices: $\gamma_{c}=\frac{\gamma_{a} \gamma_{b}-\gamma_{b} \gamma_{a}}{2}$, where $a$ is the index of the row, $b$ the index of the column, and $c$ the table entry. The only matrix commuting with all other matrices is the unit matrix $\gamma_{15}=\mathbf{1}$. 


\section{G. Real Dirac matrices and elements in coupled linear optics}

In the following we give some examples of force matrices as used in linear optics of ion beam physics [20,24]. We use the transversal coordinates $\left(x, x^{\prime}, y, y^{\prime}\right)$ as the pairs of conjugate variables $\left(q_{1}, p_{1}, q_{2}, p_{2}\right)$. In ion beam physics there is the convention to use the path length $s$ along the reference orbit as the independent variable. The force matrix of a drift (force free motion) is given by

$\dot{\psi}=\frac{d}{d s}\left(\begin{array}{c}x \\ x^{\prime} \\ y \\ y^{\prime}\end{array}\right)=\left(\begin{array}{llll}0 & 1 & 0 & 0 \\ 0 & 0 & 0 & 0 \\ 0 & 0 & 0 & 1 \\ 0 & 0 & 0 & 0\end{array}\right)\left(\begin{array}{c}x \\ x^{\prime} \\ y \\ y^{\prime}\end{array}\right)=\frac{\gamma_{0}+\gamma_{6}}{2} \psi$.

The transversal terms in a solenoid field,

$\frac{d}{d s}\left(\begin{array}{c}x \\ x^{\prime} / K \\ y \\ y^{\prime} / K\end{array}\right)=K\left(\begin{array}{cccc}0 & 1 & 1 & 0 \\ -1 & 0 & 0 & 1 \\ -1 & 0 & 0 & 1 \\ 0 & -1 & -1 & 0\end{array}\right)\left(\begin{array}{c}x \\ x^{\prime} / K \\ y \\ y^{\prime} / K\end{array}\right)$,

where $K=\frac{B_{S}}{2(B \rho)}$ with the solenoid field $B_{S}$, charge $q$, and bending radius $\rho$. The bending radius depends on the momentum $p$ and is given by

$$
\rho=\frac{p}{q B} .
$$

This can be written using RDMs as

$$
\dot{\psi}=K\left(\gamma_{0}-\gamma_{9}\right) \psi
$$

A radially focusing quadrupole is described by

$$
\frac{d}{d s}\left(\begin{array}{c}
x \\
x^{\prime} / K \\
y \\
y^{\prime} / K
\end{array}\right)=K\left(\begin{array}{cccc}
0 & 1 & 0 & 0 \\
-1 & 0 & 0 & 0 \\
0 & 0 & 0 & 1 \\
0 & 0 & 1 & 0
\end{array}\right)\left(\begin{array}{c}
x \\
x^{\prime} / K \\
y \\
y^{\prime} / K
\end{array}\right),
$$

where $K^{2}=\frac{|g|}{(B \rho)}$ with $g=\frac{\partial B_{y}}{\partial x}=\frac{\partial B_{x}}{\partial y}$ so that—written again with RDMs - one finds

$$
\dot{\psi}=K \frac{\gamma_{0}+\gamma_{1}+\gamma_{6}+\gamma_{8}}{2} \psi .
$$

The axially focusing quad is given by

$$
\dot{\psi}=K \frac{\gamma_{0}-\gamma_{1}+\gamma_{6}-\gamma_{8}}{2} \psi .
$$

A horizontal bending magnet,

$$
\frac{d}{d s}\left(\begin{array}{c}
x \\
\rho x^{\prime} \\
y \\
\rho y^{\prime}
\end{array}\right)=\frac{1}{\rho}\left(\begin{array}{cccc}
0 & 1 & 0 & 0 \\
-1 & 0 & 0 & 0 \\
0 & 0 & 0 & 1 \\
0 & 0 & 0 & 0
\end{array}\right)\left(\begin{array}{c}
x \\
\rho x^{\prime} \\
y \\
\rho y^{\prime}
\end{array}\right),
$$

expressed by RDMs,

$$
\dot{\psi}=\frac{1}{\rho} \frac{3 \gamma_{0}+\gamma_{1}+\gamma_{6}+\gamma_{8}}{4} \psi .
$$

Obviously, the scale of the momentum coordinates has to be adjusted to all individual element types and changes even with their excitation (and the beam energy), while the scale of the position coordinates is fix. The scaling transformations that are required have the following form:

$$
\left(\begin{array}{c}
a x \\
b x^{\prime} \\
a y \\
b y^{\prime}
\end{array}\right)=\left(\begin{array}{llll}
a & 0 & 0 & 0 \\
0 & b & 0 & 0 \\
0 & 0 & a & 0 \\
0 & 0 & 0 & b
\end{array}\right)\left(\begin{array}{c}
x \\
x^{\prime} \\
y \\
y^{\prime}
\end{array}\right),
$$

which allows one to scale coordinates and momenta separately. This matrix — let us call it $\mathbf{R}$ - can be written as

$$
\mathbf{R}=\frac{a+b}{2} \mathbf{1}-\frac{a-b}{2} \gamma_{3},
$$

and it is symplectic, if

$$
\begin{aligned}
\mathbf{R}^{T} \gamma_{0} \mathbf{R} & =\left(\frac{a+b}{2} \mathbf{1}-\frac{a-b}{2} \gamma_{3}\right) \gamma_{0}\left(\frac{a+b}{2} \mathbf{1}-\frac{a-b}{2} \gamma_{3}\right) \\
& =\left(\frac{(a+b)^{2}}{4}-\frac{(a-b)^{2}}{4}\right) \gamma_{0}=a b \gamma_{0} \Rightarrow a b=1,
\end{aligned}
$$

or, with the equivalent definition,

$$
\mathbf{R}=\exp \left(\gamma_{3} \varepsilon\right)=\left(\begin{array}{cccc}
e^{-\varepsilon} & 0 & 0 & 0 \\
0 & e^{\varepsilon} & 0 & 0 \\
0 & 0 & e^{-\varepsilon} & 0 \\
0 & 0 & 0 & e^{\varepsilon}
\end{array}\right) .
$$

Hence, the phase boost in the direction $\gamma_{3}$ can be understood as a transformation that changes the relative scale of the classical coordinates and momenta. The other scaling transformations are induced by the other diagonal RDMs. In the chosen basis these are $\gamma_{4}, \gamma_{12}$, and $\gamma_{15}=\mathbf{1}$. Besides $\gamma_{3}$, only $\gamma_{4}$ is a symplex and is a legitimate candidate for a symplectic scaling transformation:

$$
\mathbf{R}=\exp \left(\gamma_{4} \varepsilon\right)=\operatorname{Diag}\left(e^{-\varepsilon}, e^{\varepsilon}, e^{\varepsilon}, e^{-\varepsilon}\right)
$$

while $\gamma_{12}$ and $\gamma_{15}$ do not generate symplectic transformations:

$$
\begin{aligned}
& \exp \left(\gamma_{12} \varepsilon\right)=\operatorname{Diag}\left(e^{-\varepsilon}, e^{-\varepsilon}, e^{\varepsilon}, e^{\varepsilon}\right) \\
& \exp \left(\gamma_{15} \varepsilon\right)=\operatorname{Diag}\left(e^{\varepsilon}, e^{\varepsilon}, e^{\varepsilon}, e^{\varepsilon}\right)
\end{aligned}
$$

We note that there are symplectic transformations to independently change the scales between $q_{1}$ and $p_{1}$ and between $q_{2}$ and $p_{2}$, respectively. But there is no symplectic transformation to change the scales between the directions $\left(q_{1}, p_{1}\right)$ and $\left(q_{2}, p_{2}\right)$. Nevertheless, the rescaling that is 
required to compare force matrices of quadrupole, solenoids, dipoles, etc. to RDMs can be done by symplectic transformations, namely, by $\mathbf{R}_{3}=\exp \left(\gamma_{3} \varepsilon\right)$.

\section{DECOUPLING TWO-DIMENSIONAL HARMONIC OSCILLATORS}

Consider a system of two coupled degrees of freedom as it is common in many parts of physics. In the general case, the force matrix will be time dependent and so will be the symplectic transformation matrix $\mathbf{R}$ :

$$
\begin{aligned}
\tilde{\psi} & =\mathbf{R} \psi \\
\dot{\tilde{\psi}} & =\dot{\mathbf{R}} \psi+\mathbf{R} \dot{\psi}=(\dot{\mathbf{R}}+\mathbf{R} \mathbf{F}) \psi=\left(\dot{\mathbf{R}} \mathbf{R}^{-1}+\mathbf{R} \mathbf{F} \mathbf{R}^{-1}\right) \tilde{\psi} \\
& =\tilde{\mathbf{F}} \tilde{\psi} .
\end{aligned}
$$

Decoupling now means that the transformed force matrix

$$
\tilde{\mathbf{F}}=\dot{\mathbf{R}} \mathbf{R}^{-1}+\mathbf{R} \mathbf{F} \mathbf{R}^{-1}
$$

is either a SYSY or block diagonal. It may still be time dependent. Equation (149) can also be written as [25]

$$
\dot{\mathbf{R}}=\tilde{\mathbf{F}} \mathbf{R}-\mathbf{R} \mathbf{F} \text {. }
$$

Given that the transformation matrix has the form

$$
\mathbf{R}=\exp (-\mathbf{G} \tau)
$$

where $\mathbf{G}$ is constant, then one obtains

$$
\dot{\mathbf{R}}=-\mathbf{G R} \tilde{\mathbf{F}}=-\mathbf{G}+\mathbf{R} \mathbf{F} \mathbf{R}^{-1} .
$$

If $\tilde{\mathbf{F}}=$ const, then it follows from Eq. (152) and from the fact that $\mathbf{R}$ and $\mathbf{G}$ commute, that

$$
\begin{aligned}
\dot{\tilde{\mathbf{F}}} & =\dot{\mathbf{R}} \mathbf{F} \mathbf{R}^{-1}+\mathbf{R} \dot{\mathbf{F}} \mathbf{R}^{-1}+\mathbf{R} \mathbf{F} \dot{\mathbf{R}}^{-1}=0 \\
0 & =-\mathbf{G R} \mathbf{F} \mathbf{R}^{-1}+\mathbf{R} \dot{\mathbf{F}} \mathbf{R}^{-1}+\mathbf{R} \mathbf{F G R} \mathbf{R}^{-1} \\
\dot{\mathbf{F}} & =\mathbf{G F}-\mathbf{F G} .
\end{aligned}
$$

Obviously, the form of the Lorentz force equation [Eq. (108)] is very common in coupled linear optics and can be obtained with a few simple assumptions for symplectic transformation matrices as in Eq. (149) or for symplices as in Eq. (153) or for second moments (i.e. the $\mathbf{S}$ matrix, respectively) as in Eq. (76).

In the following we present a straightforward recipe for decoupling constant force matrices. It will be shown that this method can also be applied to transfer matriceshence, can be used to compute the properties of matched beams even in cases where the force matrix itself is not constant.

At the end of this section we sketch some problems related to the general case of time-dependent forces. But a comprehensive treatment of the time-dependent case is beyond the scope of this article.

\section{A. Eigenvalues of the force matrix}

The eigenvalues of the force matrix as expressed by Eq. (111) are given by

$$
\begin{aligned}
\lambda= & \operatorname{Diag}\left(i \omega_{1},-i \omega_{1}, i \omega_{2},-i \omega_{2}\right) \\
K_{1}= & \mathcal{E}^{2}+\vec{B}^{2}-\vec{E}^{2}-\vec{p}^{2} \\
K_{2}= & -2 \mathcal{E} \vec{p}(\vec{E} \times \vec{B})+\mathcal{E}^{2} \vec{B}^{2}+\vec{E}^{2} \vec{p}^{2}-(\vec{E} \vec{p})^{2} \\
& -(\vec{E} \vec{B})^{2}-(\vec{p} \vec{B})^{2} \\
\omega_{1}= & \sqrt{K_{1}+2 \sqrt{K_{2}}} \quad \omega_{2}=\sqrt{K_{1}-2 \sqrt{K_{2}}} \\
\operatorname{Det}(\mathbf{F})= & K_{1}^{2}-4 K_{2} .
\end{aligned}
$$

The system is stable, if the eigenvalues are purely imaginary, i.e., if the eigenfrequencies are real. This is the case, if the following conditions are fulfilled:

$$
K_{2} \geq 0 \quad K_{1} \geq 2 \sqrt{K_{2}} .
$$

The force matrix can only be a SYSY if the eigenfrequencies are equal, i.e., if $K_{2}=0$. This is for instance the case, if $\vec{E}=\vec{B}=0$. If the vectors $\vec{p}, \vec{E}$, and $\vec{B}$ are orthogonal to each other such that $\vec{p}=\alpha^{2} \vec{E} \times \vec{B}$ with some constant $\alpha$, then it follows

$$
\begin{aligned}
K_{2} & =-2 \mathcal{E} \vec{p}(\vec{E} \times \vec{B})+\mathcal{E}^{2} \vec{B}^{2}+\vec{E}^{2} \vec{p}^{2} \\
& =-2 \mathcal{E} p E B+\mathcal{E}^{2} B^{2}+E^{2} p^{2}=(\mathcal{E} B-E p)^{2},
\end{aligned}
$$

where $E=|\vec{E}|, B=|\vec{B}|$, and $p=|\vec{p}|$. In this case the force matrix is a SYSY, if $\mathcal{E} B=E p$.

We will show in the following that the standard form of the block-diagonal force matrix, as it can be obtained by symplectic transformations, is given by

$\mathbf{F}_{d}=\left(\begin{array}{cccc}0 & \alpha & 0 & 0 \\ -\beta & 0 & 0 & 0 \\ 0 & 0 & 0 & \gamma \\ 0 & 0 & -\delta & 0\end{array}\right) \quad \omega_{1}= \pm \sqrt{\alpha \beta} \quad \omega_{2}= \pm \sqrt{\gamma \delta}$.

The Hamilton matrix $\mathbf{A}=-\gamma_{0} \mathbf{F}_{d}$ corresponding to this force matrix is diagonal:

$$
\mathbf{A}=-\gamma_{0} \mathbf{F}_{d}=\operatorname{Diag}(\beta, \alpha, \delta, \gamma) .
$$

The RDM composition of $\mathbf{F}_{d}$ is given by

$$
\begin{aligned}
\mathbf{F}_{d} & =\mathcal{E} \gamma_{0}+B_{y} \gamma_{8}+p_{x} \gamma_{1}+E_{z} \gamma_{6} \\
\mathcal{E} & =\frac{\alpha+\beta+\gamma+\delta}{4} \quad B_{y}=\frac{\alpha+\beta-\gamma-\delta}{4} \\
p_{x} & =\frac{-\alpha+\beta+\gamma-\delta}{4} \quad E_{z}=\frac{\alpha-\beta+\gamma-\delta}{4} .
\end{aligned}
$$

The vectors $\vec{p}, \vec{E}$, and $\vec{B}$ are orthogonal to each other so that Eq. (156) holds, but with a reversed sign for the triple product. Obviously, one can exchange $\mathcal{E}$ with $B_{y}$ and $p_{x}$ 
with $E_{z}$ without changing the eigenfrequencies. This proves that stable systems are possible with $B_{y}>\mathcal{E}$ and/ or with $E_{z}>p_{x}$, i.e., with $|\vec{B}|>\mathcal{E}$ and/or $|\vec{E}|>|\vec{P}|$.

We define a stable system to be regular or massive, if all parameters $\alpha, \beta, \gamma$, and $\delta$ have the same sign (are positive), i.e., if the decoupled Hamiltonian function in the new coordinates $\tilde{H}=\tilde{\psi}^{T} \tilde{\mathbf{A}} \tilde{\psi}$ is positive definite:

$$
\tilde{H}=\alpha \tilde{p}_{1}^{2}+\beta \tilde{q}_{1}^{2}+\gamma \tilde{p}_{2}^{2}+\delta \tilde{q}_{2}^{2} .
$$

In a regular system the coefficients of the force matrix according to Eq. (157) yield

$$
\mathcal{E}^{2}>B_{y}^{2} \quad \mathcal{E}^{2}>p_{x}^{2} \quad \mathcal{E}^{2}>E_{z}^{2} .
$$

If one pair, either $(\alpha, \beta)$ or $(\gamma, \delta)$, is negative, then the Hamiltonian function contains a negative kinetic energy term and is indefinite. We then call the system irregular or magnetic and it is easily shown that in this case

$$
B_{y}^{2}>\mathcal{E}^{2} \quad B_{y}^{2}>p_{x}^{2} \quad B_{y}^{2}>E_{z}^{2} .
$$

\section{B. Decoupling a constant focusing channel in two dimensions}

The RDMs and the presented theory of symplectic transformations together with the EMEQ facilitates the task of decoupling - since we have already the geometrical and physical understanding that provides the optimal strategy to solve the problem. In Sec. II E we discussed the meaning of coupling and it turned out that constant symplectic force matrices (SYSYs) do not require decoupling. Instead the next derivative yields a decoupled second-order equation. In the following we would like to discuss decoupling of Eq. (18) in cases where the force matrix is not a SYSY. Then decoupling is the task to find a transformation matrix R such that

$$
\mathbf{R} \mathbf{F} \mathbf{R}^{-1}=\left(\begin{array}{cc}
\mathbf{A} & 0 \\
0 & \mathbf{B}
\end{array}\right)
$$

Since products of symplectic transformations are again symplectic, we may find the solution in several steps.

One distinguishes even and odd matrices, where odd matrices couple the 2 degrees of freedom while even ones do not [26]. In the chosen basis eight matrices, namely $\gamma_{0}$, $\gamma_{1}, \gamma_{3}, \gamma_{4}, \gamma_{6}, \gamma_{8}, \gamma_{12}, \gamma_{15}$, are even and the remaining eight matrices are odd. Since the transformation is required to be symplectic, the antisymplices $\gamma_{10}, \ldots, \gamma_{15}$ can be excluded. There are six even $\left(\gamma_{0}, \gamma_{1}, \gamma_{3}, \gamma_{4}, \gamma_{6}\right.$, and $\left.\gamma_{8}\right)$ and four odd matrices $\left(\gamma_{2}, \gamma_{5}, \gamma_{7}\right.$, and $\left.\gamma_{9}\right)$ remaining. This can directly be seen from the force matrix, which is given by

$$
\begin{aligned}
& \mathbf{F}=\mathcal{E} \gamma_{0}+\vec{p} \vec{\gamma}+\vec{E} \gamma_{0} \vec{\gamma}+\vec{B} \gamma_{14} \gamma_{0} \vec{\gamma} \\
& =\left(\begin{array}{cccc}
\mathbf{A}_{0} & -B_{z}+E_{y} & B_{x}+p_{y} \\
& -B_{x}+p_{y} & -B_{z}-E_{y} \\
E_{y}+B_{z} & B_{x}+p_{y} & \mathbf{B}_{0} \\
-B_{x}+p_{y} & B_{z}-E_{y} &
\end{array}\right) .
\end{aligned}
$$

The odd components are $p_{y}, E_{y}, B_{x}$, and $B_{z}$ corresponding to the odd matrices $\gamma_{2}, \gamma_{5}, \gamma_{7}$, and $\gamma_{9}$.

Products of even matrices result even matrices. Hence, the transformation to block-diagonal form can be done by the exclusive use of odd force matrices. Note that the odd symplices all anticommute with each other. Two of them $\left(\gamma_{2}\right.$ and $\left.\gamma_{5}\right)$ square to $+\mathbf{1}$, the other two to $-\mathbf{1}$. Hence, they are a basis of the Clifford algebra $C l(2,2)$.

Even matrices may be used for convenience, but exclusively odd matrices are required to bring a regular force matrix into block-diagonal form. The transformations have the form of Eqs. (117) and (120), where in the latter we replace the parameter $\tau$ with $\frac{\varepsilon_{b}}{2}$ :

$$
\begin{gathered}
\mathbf{R}_{b}=\exp \left(\gamma_{b} \boldsymbol{\varepsilon}_{b} / 2\right) \quad \mathbf{R}_{b}^{-1}=\exp \left(-\gamma_{b} \boldsymbol{\varepsilon}_{b} / 2\right) \\
\mathbf{F}^{\prime}=\mathbf{R}_{b} \mathbf{F} \mathbf{R}_{b}^{-1} .
\end{gathered}
$$

After each transformation, the coefficients have to be updated according to Eqs. (10) and (11).

Then the recipe for regular systems is as follows: (1) Use the rotation matrices $\mathbf{R}_{7}$ (about the $x$ axis with $\varepsilon_{7}=\arctan \frac{p_{z}}{p_{y}}$ ) and $\mathbf{R}_{9}$ (about the $z$ axis with $\varepsilon_{9}=$ $\left.-\arctan \frac{p_{x}}{p_{y}}\right)$ to align the momentum $\vec{p}$ along the $y$ axis. (2) Use $\mathbf{R}_{5}$ with $\varepsilon_{5}=-\operatorname{artanh} \frac{p_{y}}{\mathcal{E}}$ (Lorentz boost) to transform into the rest frame.

(3) Use again $\mathbf{R}_{7}$ and $\mathbf{R}_{9}$ (with $\varepsilon_{7}=\arctan \frac{B_{z}}{B_{y}}$ and $\varepsilon_{9}=$ $\left.-\arctan \frac{B_{x}}{B_{y}}\right)$ to align the magnetic field along the $y$ axis. If this is done, we have $B_{x}=B_{z}=0$. (4) Use $\mathbf{R}_{8}\left(\varepsilon_{8}=\right.$ $\left.-\arctan \frac{E_{x}}{E_{z}}\right)$ to rotate about the $y$ axis and make $E_{x}$ vanish. Note that this step is not required to obtain block-diagonal form. But it is required for a complete diagonalization.

(5) Use $\mathbf{R}_{2}\left(\varepsilon_{2}=\operatorname{artanh} \frac{E_{y}}{\mathcal{E}}\right)$ to make the parallel electric field component $E_{y}$ vanish. All but the last step should be clear. In the rest frame we have $\vec{p}=0$, so that rotation matrices can be applied without restrictions. The (odd) $\gamma_{2}$ term (i.e., $p_{y}$ ) will remain zero. The alignment of $\vec{B}$ along the $y$ axis brings the odd terms $B_{x}$ and $B_{z}$ to zero, the rotation about the $y$ axis yields $E_{x} \rightarrow 0$. The only remaining odd term is $\gamma_{5}$, i.e., $E_{y}$ :

$$
\mathbf{F}^{(0)}=m \gamma_{0}+E_{y} \gamma_{5}+E_{z} \gamma_{6}+B_{y} \gamma_{8}=\left(\begin{array}{cccc}
0 & B_{y}+E_{z}+m & E_{y} & 0 \\
-B_{y}+E_{z}-m & 0 & 0 & -E_{y} \\
E_{y} & 0 & 0 & -B y+E_{z}+m \\
0 & -E_{y} & B_{y}+E_{z}-m & 0
\end{array}\right) \text {, }
$$


where we wrote $m$ instead of $\mathcal{E}$ as the $\gamma_{0}$ coefficient to make clear that the transformation into the rest frame has already been done. The last step is required to achieve the block-diagonal form of Eq. (157), where

$$
\begin{aligned}
\mathbf{F}^{(1)} & =\tilde{m} \gamma_{0}+E_{z} \gamma_{6}+B_{y} \gamma_{8}, \quad \alpha=B_{y}+E_{z}+\tilde{m}, \\
\beta & =B_{y}-E_{z}+\tilde{m}, \quad \gamma=-B_{y}+E_{z}+\tilde{m}, \\
\delta & =-B_{y}-E_{z}+\tilde{m},
\end{aligned}
$$

where $\tilde{m}$ is given by

$$
\tilde{m}=\sqrt{m^{2}-E_{y}^{2}}
$$

This shows that phase boosts are transformations which convert electromagnetic energy into mass and vice versa in the same sense as Lorentz boosts convert mass into kinetic energy or vice versa. Note that we could also exchange the order of the last two steps, i.e., of $\mathbf{R}_{8}$ and $\mathbf{R}_{2}$. The result would be the same since $\gamma_{8}$ and $\gamma_{2}$ commute.

If the force matrix is irregular (but stable), then the energy term might be too small to perform the Lorentz boost into the rest frame as suggested by the recipe. In this case, the magnetic components provide the focusing strength that stabilize the system [see Eq. (154)].

In this case we have to prepare the force matrix in the following way.

(1) In the irregular case, we have to consider the possibility that $\vec{p}^{2} \geq \mathcal{E}^{2}$. In this case the first step is a phase rotation $\mathbf{R}_{0}$ with $\varepsilon_{0}=\frac{1}{4} \arctan \left(\frac{2 \vec{E} \vec{P}}{\vec{E}^{2}-\vec{P}^{2}}\right)$ to minimize $\vec{p}^{2}$.

(2) Align $\vec{B}$ along the $y$ axis.

(3) Rotate about the $y$ axis to maximize $p_{z}$ (i.e. to make $\left.p_{x}=0\right)$.

(4) Apply the phase boost using $\gamma_{1}$ by $\varepsilon_{1}=\frac{1}{2} \operatorname{artanh}\left(\frac{p_{z}}{B_{y}}\right)$.

If the energy is still to low, perform the following steps.

(5) Align (again) $\vec{B}$ along the $y$ axis.

(6) Rotate about the $y$ axis to maximize $E_{z}$ (i.e. to make $\left.E_{x}=0\right)$.

(7) Apply the Lorentz boost using $\gamma_{4}$ by $\varepsilon_{4}=$ $\frac{1}{2} \operatorname{artanh}\left(\frac{E_{z}}{B_{y}}\right)$. Now the energy $\mathcal{E}$ should fulfill $\mathcal{E}^{2}>\vec{p}^{2}$, so that the usual recipe for regular matrices can be applied.

An inspection of Table III may help to explain these transformations. Magnetic force matrices are irregular with respect to the definition of the state vector, i.e., the choice of $\gamma_{0}$ as explained above with Eqs. (1) and (2). If we inspect Table III, then we can see that in basis system (9), where $\gamma_{8}$ represents the energy and $\gamma_{3}$ represents $p_{x}, \gamma_{1}$ is the driver of a Lorentz boost. Interpreted in the usual system this means that it is possible to minimize $p_{z}$ using $\mathbf{R}_{1}$, if $B_{y}^{2}>p_{z}^{2}$. The second suggested transformation corresponds to the same basis and a Lorentz boost along $y$ [accordingly in basis system (9), this is driven by $\gamma_{4}$ ]. The fact that we have to look at a different basis system to find the appropriate symplectic transformation, legitimizes us to claim that such systems are irregular. The examples given in Sec. III G have $\gamma_{0}$ coefficients which are at least as strong as any other component. This fact seems to support the assumption that only regular systems exist in linear coupled optics. This assumption is wrong and in the following section we give an example for an irregular system.

\section{Example for an irregular system}

In an accompanying paper we describe a simplified and idealized cyclotron model with space charge, which is an example for an irregular system [12]. The force matrix can easily be transformed into the form of Eq. (167). Using $\psi=\left(x, x^{\prime}, l, \delta\right)^{T}$ as the canonical coordinates, where $x$ and $l$ are the horizontal and longitudinal position of the ion, $x^{\prime}=\frac{d x}{d s}$ is the horizontal angle and $\delta=\frac{p-p_{0}}{p_{0}}$ is the relative deviation of the momentum $p$ from the average momentum $p_{0}$. The (constant) force matrix $\mathbf{F}$ is given by

$$
\mathbf{F}=\left(\begin{array}{cccc}
0 & 1 & 0 & 0 \\
-k_{x}+K_{x} & 0 & 0 & h \\
-h & 0 & 0 & \frac{1}{\gamma^{2}} \\
0 & 0 & K_{z} \gamma^{2} & 0
\end{array}\right)
$$

where $\gamma$ is the relativistic factor, $h=1 / r$ is the inverse bending radius of the magnetic field, and $k_{x}=$ $h^{2}(1+n)=h^{2} \gamma^{2}$ is the horizontal restoring force. $n=$ $\frac{r}{B} \frac{d B}{d r}$ is the field index. $K_{x}$ and $K_{z}$ are the horizontal and axial space charge force [12], respectively. The eigenfrequencies of the force matrix are

$$
\begin{aligned}
a \equiv \frac{k_{x}-K_{x}-K_{z}}{2} & b \equiv K_{z}\left(K_{x}+h^{2} \gamma^{2}-k_{x}\right) \\
\Omega=\sqrt{a+\sqrt{a^{2}-b}} & \omega=\sqrt{a-\sqrt{a^{2}-b}} .
\end{aligned}
$$

The RDM coefficients are

$$
\begin{aligned}
\mathcal{E} & =\frac{1}{4}\left(1+k_{x}-K_{x}+\frac{1}{\gamma^{2}}-\gamma^{2} K_{z}\right) \\
P_{x} & =\frac{1}{4}\left(-1+k_{x}-K_{x}+\frac{1}{\gamma^{2}}+\gamma^{2} K_{z}\right) \\
P_{y} & =P_{z}=0 \quad E_{x}=B_{x}=0 \quad E_{y}=B_{z}=-\frac{h}{2} \\
E_{z} & =\frac{1}{4}\left(1-k_{x}+K_{x}+\frac{1}{\gamma^{2}}+\gamma^{2} K_{z}\right) \\
B_{y} & =\frac{1}{4}\left(1+k_{x}-K_{x}-\frac{1}{\gamma^{2}}+\gamma^{2} K_{z}\right) .
\end{aligned}
$$

The system is reasonably simple as there are only two elements that are not block diagonal. This allows one to guess a transformation matrix instead of using the above recipe: 


$$
\begin{aligned}
\mathbf{R} & =\exp \left((r+1 / r) \frac{s}{2} \gamma_{2}+(r-1 / r) \frac{s}{2} \gamma_{7}\right) \\
& =\cosh (s) \mathbf{1}+\left[(r+1 / r) \gamma_{2}+(r-1 / r) \gamma_{7}\right] \frac{\sinh (s)}{2},
\end{aligned}
$$

where the following abbreviations are used:

$$
\begin{aligned}
A & =\frac{h}{\Omega^{2}+K_{z}} \quad B=\frac{h}{\omega^{2}+K_{z}} \quad \cosh (s)=\sqrt{\frac{B}{B-A}} \\
\sinh (s) & =-\sqrt{\frac{A}{B-A}} \quad r=\frac{1}{\sqrt{K_{z}} \gamma} .
\end{aligned}
$$

The transformed force matrix is then

$$
\begin{aligned}
& \tilde{\mathbf{F}}=\mathbf{R F R}^{-1}=\left(\begin{array}{cccc}
0 & 1 & 0 & 0 \\
-\beta & 0 & 0 & 0 \\
0 & 0 & 0 & -\Gamma \\
0 & 0 & \gamma^{2} K_{z} & 0
\end{array}\right) \\
& \beta=\frac{A K_{z}+B\left(k_{x}-K_{x}\right)-2 h}{B-A} \quad \Gamma=\frac{B K_{z}+A\left(k_{x}-K_{x}\right)-2 h}{(B-A) \gamma^{2} K_{z}} .
\end{aligned}
$$

Since $\gamma^{2} K_{z}>0$ and $\Gamma>0$ is given, the transformed force matrix is irregular and the transformed Hamiltonian $\tilde{H}$ has the form

$$
\tilde{H}=\tilde{x}^{\prime 2}+\beta \tilde{x}^{2}-\Gamma \tilde{\delta}^{2}-\gamma^{2} K_{z} l^{2} .
$$

It is also an example for a classical system, that cannot be diagonalized by the method of "symplectic rotation" of Teng and Edwards [12].

\section{Diagonalization}

Though the force matrix has been decoupled, there is motivation to continue the process of diagonalization by a few more transformations, since then the product of the transformation matrices equals the matrix of eigenvectors and the resulting diagonal matrix contains the eigenvalues. According to Eqs. (56), (57), (82), and (85), the matched beam ellipse can then be directly computed for any combination of the emittances $\varepsilon_{1}$ and $\varepsilon_{2}$.

The intention is to bring $\mathbf{F}$ into the form of Eq. (56). Since we expect imaginary eigenvalues, the transformation matrices cannot be real symplectic. The first "direction" that we use is $i \gamma_{6}$, which can formally be identified with a Lorentz boost with an infinite imaginary relative "velocity," so that the "angle" is given by

$$
\varepsilon=\lim _{\beta \rightarrow \infty} \operatorname{artanh}(i \beta) / 2=\lim _{\beta \rightarrow \infty} \frac{i}{2} \arctan (\beta)=i \frac{\pi}{4} .
$$

The matrix is explicitly given by

$$
\mathbf{P}_{6}=\exp \left(i \gamma_{6} \frac{\pi}{4}\right)=\frac{1}{\sqrt{2}}\left(\begin{array}{cccc}
1 & -i & 0 & 0 \\
-i & 1 & 0 & 0 \\
0 & 0 & 1 & -i \\
0 & 0 & -i & 1
\end{array}\right)
$$

This matrix has some remarkable properties: It is unitary, symmetric, and symplectic. The transformed force matrix is

$$
\begin{aligned}
\mathbf{F}^{(2)} & =\mathbf{P}_{6} \mathbf{F}^{(5)} \mathbf{P}_{6}^{-1} \\
& =\left(\begin{array}{cccc}
i\left(B_{y}+\tilde{m}\right) & E_{z} & 0 & 0 \\
E_{z} & -i\left(B_{y}+\tilde{m}\right) & 0 & 0 \\
0 & 0 & -i\left(B_{y}-\tilde{m}\right) & E_{z} \\
0 & 0 & E_{z} & i\left(B_{y}-\tilde{m}\right)
\end{array}\right),
\end{aligned}
$$

and the final transformation includes two RDMs, $\gamma_{0}$ and $\gamma_{8}$. This combination allows for a relative phase rotation between both degrees of freedom as in Eq. (127), but now with imaginary angles:

$$
\begin{aligned}
\mathbf{P}_{8} & =\exp \left(i \gamma_{0} \frac{\varepsilon_{1}-\varepsilon_{2}}{2}+i \gamma_{8} \frac{\varepsilon_{1}+\varepsilon_{2}}{2}\right) \\
& =\left(\begin{array}{cccc}
c_{1} & i s_{1} & 0 & 0 \\
-i s_{1} & c_{1} & 0 & 0 \\
0 & 0 & c_{2} & -i s_{2} \\
0 & 0 & i s_{2} & c_{2}
\end{array}\right),
\end{aligned}
$$

where

$$
\begin{aligned}
c_{i} & =\cosh \varepsilon_{i} \quad s_{i}=\sinh \varepsilon_{i} \\
\varepsilon_{1} & =\frac{1}{2} \operatorname{Artanh}\left(\frac{E_{z}}{B_{y}+\tilde{m}}\right) \quad \varepsilon_{2}=\frac{1}{2} \operatorname{Artanh}\left(\frac{E_{z}}{B_{y}-\tilde{m}}\right),
\end{aligned}
$$

so that

$$
\begin{aligned}
& \mathbf{F}^{(3)}=\mathbf{P}_{8} \mathbf{F}^{(6)} \mathbf{P}_{8}^{-1}=\lambda=\left(\begin{array}{cccc}
i\left(B_{y}+\tilde{m}\right) a & 0 & 0 & 0 \\
0 & -i\left(B_{y}+\tilde{m}\right) a & 0 & 0 \\
0 & 0 & -i\left(B_{y}-\tilde{m}\right) b & 0 \\
0 & 0 & 0 & i\left(B_{y}-\tilde{m}\right) b
\end{array}\right) \\
& a=\sqrt{1-\frac{E_{z}^{2}}{\left(B_{y}+\tilde{m}\right)^{2}}}=\sqrt{\frac{4 \alpha \beta}{(\alpha+\beta)^{2}}} \quad b=\sqrt{1-\frac{E_{z}^{2}}{\left(B_{y}-\tilde{m}\right)^{2}}}=\sqrt{\frac{4 \delta \gamma}{(\delta+\gamma)^{2}}}
\end{aligned}
$$


In terms of RDMs the diagonalized force matrix is

$$
\begin{aligned}
\mathbf{F}^{(3)}= & -\frac{i}{2}\left[a\left(B_{y}+\tilde{m}\right)-b\left(B_{y}-\tilde{m}\right)\right] \gamma_{3} \\
& -\frac{i}{2}\left[a\left(B_{y}+\tilde{m}\right)+b\left(B_{y}-\tilde{m}\right)\right] \gamma_{4} .
\end{aligned}
$$

Note that the last (double) transformations is not necessary, if $E_{z}$ is already zero. The eigenvectors are now given by the product of all transformation matrices (in their order).

\section{E. Summary: Decoupling and diagonalization}

The force matrix $\mathbf{F}$ of stable systems was shown to have the form of Eq. (56) where the diagonal matrix $\lambda$ has the form of Eq. (59). It turned out that there is an intermediate stage in the process which corresponds to a diagonalized Hamiltonian function. The similarity transformation $\mathbf{R}$ to reach this intermediate stage is symplectic and with Eqs. (17) and (18) it follows that

$$
\begin{aligned}
\mathbf{A}_{d} & =\operatorname{Diag}(\beta, \alpha, \delta, \gamma) \quad \mathbf{F}_{d}=\mathbf{R} \mathbf{F} \mathbf{R}^{-1} \\
\gamma_{0} \mathbf{A}_{d} & =\mathbf{R} \gamma_{0} \mathbf{A} \mathbf{R}^{-1}=-\mathbf{R} \gamma_{0} \mathbf{A} \gamma_{0} \mathbf{R}^{T} \gamma_{0} \\
\mathbf{A}_{d} & =\gamma_{0} \mathbf{R} \gamma_{0} \mathbf{A} \gamma_{0} \mathbf{R}^{T} \gamma_{0} .
\end{aligned}
$$

This equation can be reversed by multiplication with $\mathbf{R}$ $\left(\mathbf{R}^{T}\right)$ from the right (left), respectively:

$$
\mathbf{A}=\mathbf{R}^{T} \mathbf{A}_{d} \mathbf{R}
$$

Note that $\mathbf{R}$ is in general not orthogonal, i.e., $\mathbf{R}^{T} \mathbf{R} \neq \mathbf{1}$. Hence, a symplectic diagonalization can usually not be replaced by an orthogonal transformation. Nevertheless the symplectic decoupling diagonalizes the Hamiltonian.

\section{F. The RDM coefficients of the transfer matrix and the tunes}

In the following we use the knowledge of the matrix of eigenvectors $\mathbf{E}$ to further investigate the general structure of the transfer matrix using Eqs. (66) and (69). The "amplitudes" of $\mathbf{M}_{s}$ are limited by the sine function, but the structure is identical to the structure of the force matrix, i.e., $\mathbf{M}_{s}$ is composed of symplices. The structural difference between transfer and force matrix is given by $\mathbf{M}_{c}$.

Note that the decoupling algorithm of Teng and Edwards for two-dimensional systems is applied to the transfer matrix instead of the force matrix. But from Eqs. (63) and (66) it is clear that the computation of the eigenvectors does not require to include $\mathbf{M}_{c}$. The decoupling can therefore be done with the force matrix $\mathbf{F}$, - which is favorable in case of constant forces-or with $\mathbf{M}_{s}$. The latter enables the treatment of periodic systems with varying force matrix F. If the decoupling algorithm is applied to $\mathbf{M}_{s}$, then one obtains by comparison of Eqs. (66), (182), and (167):

$$
\begin{gathered}
\Sigma_{c}=\frac{1}{4} \operatorname{Tr}(\mathbf{M}) \quad \Sigma_{s}=\frac{a(\alpha+\beta)+b(\gamma+\delta)}{4} \\
\Delta_{s}=\frac{a(\alpha+\beta)-b(\gamma+\delta)}{4} .
\end{gathered}
$$

The last missing value to determine the coupled tunes is $\Delta_{c}$. We will argue in the following that the decoupling of $\mathbf{M}_{s}$ will casually diagonalize $\mathbf{M}_{c}$ and, hence, yields $\Delta_{c}$.

It is noteworthy that $\gamma_{12}$ is the only even matrix that anticommutes with all odd symplices (see Table V). This means that $\gamma_{12}$ plus the odd symplices $\gamma_{2}, \gamma_{5}$, and $\gamma_{7}$ build an alternative basis of $\mathrm{Cl}(3,1)$-in which $\gamma_{9}$ is the pseudoscalar (see Table III).

In the following we use the diagonalization steps and reconstruct the components of $\mathbf{M}_{c}$. The scalar part can be ignored as it cannot be transformed. The interesting part therefore is $\mathbf{E} \gamma_{12} \mathbf{E}^{-1}$. The matrix $\mathbf{E}$ is the reverse product of all transformation matrices and, hence, we may analyze the structure of $\mathbf{M}_{c}$ by applying all steps of the diagonalization in reversed order.

As can be seen in Table IV, the matrix $\gamma_{12}$ commutes with $\gamma_{0}, \gamma_{6}$, and $\gamma_{8}$, so that the reversed transformations of Eqs. (178) and (181) have no effect on $\gamma_{12}$. Hence, we can skip the diagonalization steps and go back to the transformations of Sec. IV B. The general structure of the resulting terms can be seen from Table IV. All transformations are symplectic, i.e., are launched by symplices. All nonvanishing commutators of RDMs in the range $\gamma_{10}, \ldots, \gamma_{14}$ with symplices yield matrices in the range $\gamma_{10}, \ldots, \gamma_{14}$. Therefore the symplectic (back) transformation of $\gamma_{12}$ exclusively yields axial-vector and pseudoscalar components. In the following we assume that the one-turn-transfer matrix is known and "off resonance," i.e., none of the coefficients of Eq. (63) are zero.

Then the first step of the back transformation is

$$
\mathbf{X}^{(1)}=\mathbf{R}_{2}^{-1} \gamma_{12} \mathbf{R}_{2}=\cosh \varepsilon_{2} \gamma_{12}+\sinh \varepsilon_{2} \gamma_{14} .
$$

The second step-the rotation about the $y$ axis using $\gamma_{8}$ again has no effect, since both $\gamma_{12}$ and $\gamma_{14}$ commute with $\gamma_{8}$. Hence, the next steps are the rotations about the $z$ and the $x$ axis. Both rotations commute with $\gamma_{14}$-so that the second term in Eq. (186) remains unchanged. But the first term is an (axial) vector term which rotates analogue to the vector term $\gamma_{2}$. Hence, the rotations create coefficients of $\gamma_{11}$ and $\gamma_{13}$. The next step is the inverse Lorentz boost in the $y$ direction with $\gamma_{5}$. Since $\gamma_{5}$ commutes with $\gamma_{11}$ and $\gamma_{13}$, only the $\gamma_{12}$ term transforms and launches a $\gamma_{10}$ component.

The remaining last two transformations are again rotations about the $x$ and the $z$ axis. Rotations will neither change the pseudoscalar coefficient nor the timelike axial vector component $\gamma_{10}$. Hence, the pseudoscalar coefficient of the transfer matrix can be used to determine if a phase boost is required for decoupling. According to the 
TABLE V. Anticommutator table of the group of $\gamma$ matrices: $\gamma_{c}=\frac{\gamma_{a} \gamma_{b}+\gamma_{b} \gamma_{a}}{2}$, where $a$ is the index of the row, $b$ the index of the column, and $c$ the table entry.

\begin{tabular}{|c|c|c|c|c|c|c|c|c|c|c|c|c|c|c|c|c|}
\hline & 0 & 1 & 2 & 3 & 4 & 5 & 6 & 7 & 8 & 9 & 10 & 11 & 12 & 13 & 14 & 15 \\
\hline 0 & -15 & & & & & & & 11 & 12 & 13 & & -7 & -8 & -9 & & 0 \\
\hline 1 & & 15 & & & & -13 & 12 & 10 & & & 7 & & 6 & -5 & & 1 \\
\hline 2 & & & 15 & & 13 & & -11 & & 10 & & 8 & -6 & & 4 & & 2 \\
\hline 3 & & & & 15 & -12 & 11 & & & & 10 & 9 & 5 & -4 & & & 3 \\
\hline 4 & & & 13 & -12 & 15 & & & 14 & & & & & -3 & 2 & 7 & 4 \\
\hline 5 & & -13 & & 11 & & 15 & & & 14 & & & 3 & & -1 & 8 & 5 \\
\hline 6 & & 12 & -11 & & & & 15 & & & 14 & & -2 & 1 & & 9 & 6 \\
\hline 7 & 11 & 10 & & & 14 & & & -15 & & & -1 & -0 & & & -4 & 7 \\
\hline 8 & 12 & & 10 & & & 14 & & & -15 & & -2 & & -0 & & -5 & 8 \\
\hline 9 & 13 & & & 10 & & & 14 & & & -15 & -3 & & & -0 & -6 & 9 \\
\hline 10 & & 7 & 8 & 9 & & & & -1 & -2 & -3 & -15 & & & & & 10 \\
\hline 11 & -7 & & -6 & 5 & & 3 & -2 & -0 & & & & 15 & & & & 11 \\
\hline 12 & -8 & 6 & & -4 & -3 & & 1 & & -0 & & & & 15 & & & 12 \\
\hline 13 & -9 & -5 & 4 & & 2 & -1 & & & & -0 & & & & 15 & & 13 \\
\hline 14 & & & & & 7 & 8 & 9 & -4 & -5 & -6 & & & & & -15 & 14 \\
\hline 15 & 0 & 1 & 2 & 3 & 4 & 5 & 6 & 7 & 8 & 9 & 10 & 11 & 12 & 13 & 14 & 15 \\
\hline
\end{tabular}

decoupling recipe this is the case, if (and only if) the EMEQ components of the force matrix fulfill $\vec{E} \vec{B} \neq 0$.

Hence one finds

$$
\begin{aligned}
-\sinh \varepsilon_{2} \Delta_{c} & =\gamma_{14} \cdot \mathbf{M} \\
\tilde{\mathbf{X}} & =\mathbf{M}_{c}-\frac{1}{4} \operatorname{Tr}\left(\mathbf{M}_{c}\right)=-\Delta_{c} \mathbf{E} \gamma_{12} \mathbf{E}^{-1} .
\end{aligned}
$$

If the decoupling transformation has been computed, then

$$
\Delta_{c}=-\gamma_{12} \mathbf{E}^{-1}\left[\mathbf{M}_{c}-\frac{1}{4} \operatorname{Tr}\left(\mathbf{M}_{c}\right)\right] \mathbf{E} .
$$

Hence, we may now solve Eq. (63) for the tunes:

$$
\tan (\bar{\omega} \tau)=\frac{\Sigma_{s}}{\Sigma_{c}}=-\frac{\Delta_{c}}{\Delta_{s}} \quad \tan (\Delta \omega \tau)=\frac{\Delta_{s}}{\Sigma_{c}}=\frac{\Delta_{c}}{\Sigma_{s}} .
$$

If $\tau$ is the repetition time (or length of the design orbit) of the accelerator, then the tune $Q_{i}$ equals $Q_{i}=\frac{\omega_{i} \tau}{2 \pi}$.

Note that the anticommutators $\frac{\gamma_{\mu} \gamma_{\nu}+\gamma_{\nu} \gamma_{\mu}}{2}$ for $\mu, \nu \in$ $[10, \ldots, 14]$ form a diagonal $5 \times 5$ matrix and, hence, are the basis of a five-dimensional phase space (see Table V).

\section{G. Transformation to equal frequencies}

Consider now a time-dependent symplectic transformation as derived in Eq. (152) with

$$
\mathbf{G}=i \omega \gamma_{4},
$$

with the corresponding matrices

$$
\begin{aligned}
\mathbf{U} & =e^{i \omega \gamma_{4} \tau}=\operatorname{Diag}\left(e^{-i \omega \tau}, e^{i \omega \tau}, e^{-i \omega \tau}, e^{i \omega \tau}\right) \\
\mathbf{U}^{-1} & =e^{-i \omega \gamma_{4} \tau}=\operatorname{Diag}\left(e^{i \omega \tau}, e^{-i \omega \tau}, e^{i \omega \tau}, e^{-i \omega \tau}\right),
\end{aligned}
$$

which does not affect the diagonalized force matrix $\mathbf{F}$ :

$$
\mathbf{U} \mathbf{F} \mathbf{U}^{-1}=\mathbf{F},
$$

but results in a frequency shift such that

$$
\begin{gathered}
\mathbf{F}^{\prime}=\mathbf{F}+i \omega \gamma_{4}=\left(\begin{array}{cccc}
i \Omega_{1} & 0 & 0 & 0 \\
0 & -i \Omega_{1} & 0 & 0 \\
0 & 0 & i \Omega_{2} & 0 \\
0 & 0 & 0 & -i \Omega_{2}
\end{array}\right) \\
\Omega_{1}=\left(\tilde{m}+B_{y}\right) a-\omega \\
\Omega_{2}\left(\tilde{m}-B_{y}\right) b+\omega .
\end{gathered}
$$

One may now chose $\omega$ such that $\Omega_{1}=\Omega_{2}=\Omega$; that is,

$$
\begin{aligned}
\left(\tilde{m}+B_{y}\right) a-\omega & =\left(\tilde{m}-B_{y}\right) b+\omega \\
2 \omega & =\left(\tilde{m}+B_{y}\right) a-\left(\tilde{m}-B_{y}\right) b \\
\omega & =\frac{\tilde{m}(a-b)+B_{y}(a+b)}{2}
\end{aligned}
$$

then we obtain the result that

$$
\mathbf{F}=-i \Omega \gamma_{3}
$$

It is also possible to choose

$$
\mathbf{G}=i \omega \gamma_{3},
$$

resulting in a frequency shift such that

$$
\begin{gathered}
\mathbf{F}^{\prime}=\mathbf{F}+i \omega \gamma_{3}=-i \Omega \gamma_{4} \quad \omega=\frac{\tilde{m}(a+b)+B_{y}(a-b)}{2} \\
\Omega=\left(\tilde{m}+B_{y}\right) a-\omega .
\end{gathered}
$$




\section{H. Time-dependent forces}

The treatment of time-dependent symplectic transformations goes beyond the scope of this article. Nevertheless we would like to make a few comments.

If the parameter $\varepsilon$ of Eq. (120) is time dependent, it follows that

$$
\dot{\mathbf{R}}_{b} \mathbf{R}_{b}^{-1}=\gamma_{b} \dot{\varepsilon} .
$$

Equation (149) can then be written as

$$
\tilde{\mathbf{F}}=\gamma_{b} \dot{\varepsilon}+\mathbf{R}_{b} \mathbf{F} \mathbf{R}_{b}^{-1} .
$$

Consider for instance the case of a force matrix $\mathbf{F}$ with the simple periodic form

$$
\mathbf{F}=\mathbf{F}_{0}[1+\alpha \cos (\omega \tau)]
$$

Then the use of a symplectic transformation matrix

$$
\mathbf{R}=\exp \left[-\alpha / \omega \mathbf{F}_{0} \sin (\omega \tau)\right]
$$

results in a constant force matrix:

$\dot{\tilde{\psi}}=\left[-\alpha \mathbf{F}_{0} \cos (\omega \tau)+\mathbf{F}_{0}+\mathbf{F}_{0} \alpha \cos (\omega \tau)\right] \tilde{\psi}=\mathbf{F}_{0} \tilde{\psi}$.

The general time-dependent case is more involved. If we intend to use $\mathbf{R}_{b}$ in order to let the corresponding odd component of the force matrix vanish, then Eq. (199) implies that we have to take the new (odd) term $\gamma_{b} \dot{\varepsilon}$ into account.

Following the recipe from above, the first step is the alignment of the momentum along the $y$ axis using $\mathbf{R}_{7}$ and $\mathbf{R}_{9}$, respectively. This introduces new (time-dependent) terms in the coefficients of $\gamma_{7}$ and $\gamma_{9}$-which is no problem at this stage. The second step is a boost along the $y$ axis using $\gamma_{5}$ with the intention to get rid of $p_{y}$. Even this is not a problem, since $\gamma_{5}$ corresponds to $E_{y}$, which has not yet been considered. Hence, it is always possible to transform into the "rest frame" at the cost of additional "field" terms in $B_{x}, B_{z}$, and $E_{y}$.

Nevertheless, the next steps include difficulties, since the alignment of $\vec{B}$ along the $y$ axis induces again additional terms in $B_{x}, B_{z}$. If the generators of the transformation and the terms to act on are identical, the straightforward approach fails. The rotation about the $x$ axis

$$
\begin{aligned}
\varepsilon & =\arctan \left(\frac{B_{z}}{B_{y}}\right) \quad \dot{\varepsilon}=\frac{\dot{B}_{z} B_{y}-\dot{B}_{y} B_{z}}{B_{y}^{2}+B_{z}^{2}} \\
B_{x} & \rightarrow B_{x}+\frac{\dot{B}_{z} B_{y}-\dot{B}_{y} B_{z}}{B_{y}^{2}+B_{z}^{2}} .
\end{aligned}
$$

More general one has

$$
\begin{aligned}
& \varepsilon=\arctan \left(\frac{X}{Y}\right) \Rightarrow \dot{\varepsilon}=\frac{\dot{X} Y-\dot{Y} X}{Y^{2}+X^{2}} \\
& \varepsilon=\operatorname{artanh}\left(\frac{X}{Y}\right) \Rightarrow \dot{\varepsilon}=\frac{\dot{X} Y-\dot{Y} X}{Y^{2}-X^{2}} .
\end{aligned}
$$

\section{SUMMARY}

We introduced the real Dirac matrices and the concept of the symplex in two-dimensional coupled linear optics, i.e., classical Hamiltonian mechanics. Since these matrices form a basis for all real-valued $4 \times 4$ matrices, we can claim that our survey of symplectic transformations for such systems is complete and final. We have shown that a subset of these transformations is isomorphic to rotations and Lorentz boost as applied to Dirac spinors in quantum electrodynamics. We used this isomorphism to describe a general and straightforward decoupling algorithm for the case of constant forces and for transfer matrices in the context of periodic motion. The algorithm was tested numerically using random force matrices of stable systems and it never failed in the case of stable systems.

There are six possible choices for the symplectic unit matrix $\gamma_{0}$ as listed in Table III corresponding to six antisymmetric RDMs. These choices are nothing but permutations of the dynamical variables in the state vector, and hence they do not differ in their physical content. For each possible $\gamma_{0}$ there are two choices for $\gamma_{1}, \gamma_{2}$, and $\gamma_{3}$, which can be transformed into each other by the phase rotation. Hence, the choice of the system of RDMs is unique as soon as the order of the canonical coordinates and momenta is fixed.

We clarified the structure of the transfer matrix in twodimensional coupled linear optics which we believe is the first universally valid generalization of the Courant-Snyder theory.

\section{DISCUSSION AND OUTLOOK}

Since the Dirac matrices are usually known in the context of relativistic quantum electrodynamics (QED), it is sort of inevitable to contemplate the relationship between QED and classical mechanics. It is known that the eigenvalues of stable systems lie on the unit circle in the complex plane [18], so that the treatment of eigenvectorswhich are essential to the theory-requires the introduction of imaginary numbers. David Hestenes gave a geometrical interpretation of the unit imaginary in quantum mechanics $[27,28]$. In the context of two-dimensional symplectic flow, the unit imaginary can be interpreted statistically, as the complex eigenvectors are able to replace a statistical ensemble of real-valued trajectories. This is to say that the matrix $\sigma=\psi \bar{\psi}$ with a real-valued spinor $\psi$ has a vanishing determinant, i.e., zero emittance-unless we introduce some kind of averaging - either over a statistical ensemble or over time. The introduction of complex-valued eigenvectors circumvents this problem as well known in the Courant-Snyder theory.

There is a theorem that all symplectic systems of the same dimension are isomorphic [18]. If the reverse is also true then the existence of the EMEQ suggests that Minkowski space-time is "embedded" in a four-dimensional phase space and that special relativity can be "deduced" from classical symplectic motion. This deduction implies that 
the components of the relativistic momentum are not proper canonical variables in the classical sense, but instead energy and momentum appear to be second moments of the dynamical variables of a two-dimensional symplectic system. In fact, the invariance of mass is then formally and physically identical to the invariance of the phase-space volume (emittance) in symplectic flow. This formal analogy seems to find its correspondence in the relation

$$
E=m c^{2}=\hbar \omega
$$

There are still many open questions as, for instance: why are there six field components in electromagnetic theory instead of ten as we should expect from two-dimensional symplectic motion? The answer could be related to the dimensionality of the system under consideration. The phase-space dimension $n$ of a classical system and the number $\nu$ of "valid" symplices are related by Eq. (23) and can be resolved for $n$ :

$$
n=\sqrt{2 \nu+\frac{1}{4}}-\frac{1}{2} .
$$

If we insert $\nu=6$, then we find $n=3$. Classically the phase-space dimension should be an even number, since Hamilton mechanics is based on pairs $\left(q_{i}, p_{i}\right)$. But a generalization of Hamilton mechanics for odd phase-space dimensions has already been presented by Nambu utilizing several distinct Hamilton functions [29]. We believe that the description of odd-dimensional phase spaces can also be done using canonical pairs $\left(q_{i}, p_{i}\right)$-if the dimensionality of a system is defined by the number of available force components, i.e., the number of symplices. Relativistic electromagnetic theory knows six symplices (and, hence, six symplectic transformations), two-dimensional coupled optics has ten, in Appendix A we argue that complexification of the presented theory results in 15 valid symplices corresponding to a five-dimensional phase space.

\section{ACKNOWLEDGMENTS}

MATHEMATICA ${ }^{\circledR} 5.2$ has been used for some of the symbolic calculations. Addition software has been written in " $\mathrm{C}$ " and been compiled with the GNU(C-C++ compiler 3.4.6 on Scientific Linux.

\section{APPENDIX A: COMPLEXIFICATION}

If we consider complex spinors then it is quickly shown that the Hamilton equations are

$$
\begin{aligned}
& \psi_{i}=\frac{q_{i}+i p_{i}}{\sqrt{2}} \quad \dot{q}_{i}=\frac{i}{\sqrt{2}}\left(\frac{\partial H}{\partial \psi_{i}}-\frac{\partial H}{\partial \psi_{i}^{\star}}\right) \\
& \dot{p}_{i}=-\frac{1}{\sqrt{2}}\left(\frac{\partial H}{\partial \psi_{i}}+\frac{\partial H}{\partial \psi_{i}^{\star}}\right) \quad i \dot{\psi}_{i}=\frac{\partial H}{\partial \psi_{i}^{\star}} \quad i \dot{\psi}_{i}^{\star}=-\frac{\partial H}{\partial \psi_{i}}
\end{aligned}
$$

Given the Hamiltonian function is expressed by

$$
H=\psi^{\dagger} \mathcal{A} \psi=\sum_{i, j} \psi_{i}^{\star} \mathcal{A}_{i j} \psi_{j}
$$

with the matrix $\mathcal{A}$ then one obtains

$$
\begin{aligned}
& i \dot{\psi}_{k}=\frac{\partial H}{\partial \psi_{k}^{\star}}=\sum_{i, j} \delta_{i k} \mathcal{A}_{i j} \psi_{j}=\sum_{j} \mathcal{A}_{k j} \psi_{j} \\
& i \dot{\psi}_{k}^{\star}=-\frac{\partial H}{\partial \psi_{k}}=-\sum_{i, j} \psi_{i}^{\star} \mathcal{A}_{i j} \delta_{j k}=-\sum_{i} \psi_{i}^{\star} \mathcal{A}_{i k} .
\end{aligned}
$$

With implicit summation we write

$$
i \dot{\psi}=\mathcal{A} \psi \quad i \dot{\psi}^{\dagger}=-\psi^{\dagger} \mathcal{A} \text {. }
$$

If we take the adjunct of the second equation

$$
i \dot{\psi}=\left(\mathcal{A}^{T}\right)^{\star} \psi=\mathcal{A}^{\dagger} \psi,
$$

it becomes obvious that the matrix $\mathcal{A}$ must be self-adjoint or Hermitian. If we switch back to the $\left(q_{i}, p_{i}\right)$ approach with $\mathcal{A}=\mathbf{A}+i \mathbf{B}, \mathbf{A}=\mathbf{A}^{T}, \mathbf{B}^{T}=-\mathbf{B}$,

$H=\psi^{\dagger} \mathcal{A} \psi \quad H=\frac{1}{2}(q-i p)^{T}(\mathbf{A}+i \mathbf{B})(q+i p)$

$H=\frac{1}{2}\left(q^{T} \mathbf{A} q+p^{T} \mathbf{A} p-2 q^{T} \mathbf{B} p\right)$,

then we find additional restrictions compared to a classical system: The matrix $\mathbf{A}$ appears for both-coordinates and momenta. The complex notation of a four-dimensional spinor is therefore not suitable for the general description of four-dimensional classical harmonic oscillators.

The symmetric matrix $\mathbf{A}$ in the real-valued twodimensional description has been replaced by a Hermitian matrix in the complex notation. It can be shown that the generalization of a symplectic transformation matrix $\mathbf{U}$ then is

$$
\gamma_{0}=\mathbf{U} \gamma_{0} \mathbf{U}^{\dagger}
$$

There are 15 complex Dirac matrices with zero trace that obey Eq. (A7) so that the phase space is, according to Eq. (206), five dimensional.

\section{APPENDIX B: THE $\boldsymbol{\gamma}$ MATRICES}

To complete the list of the real $\gamma$ matrices used throughout this paper, 


$$
\begin{aligned}
& \gamma_{4}=\left(\begin{array}{cccc}
-1 & 0 & 0 & 0 \\
0 & 1 & 0 & 0 \\
0 & 0 & 1 & 0 \\
0 & 0 & 0 & -1
\end{array}\right) \quad \gamma_{5}=\left(\begin{array}{cccc}
0 & 0 & 1 & 0 \\
0 & 0 & 0 & -1 \\
1 & 0 & 0 & 0 \\
0 & -1 & 0 & 0
\end{array}\right) \\
& \gamma_{6}=\left(\begin{array}{llll}
0 & 1 & 0 & 0 \\
1 & 0 & 0 & 0 \\
0 & 0 & 0 & 1 \\
0 & 0 & 1 & 0
\end{array}\right) \quad \gamma_{7}=\left(\begin{array}{cccc}
0 & 0 & 0 & 1 \\
0 & 0 & -1 & 0 \\
0 & 1 & 0 & 0 \\
-1 & 0 & 0 & 0
\end{array}\right) \\
& \gamma_{8}=\left(\begin{array}{cccc}
0 & 1 & 0 & 0 \\
-1 & 0 & 0 & 0 \\
0 & 0 & 0 & -1 \\
0 & 0 & 1 & 0
\end{array}\right) \quad \gamma_{9}=\left(\begin{array}{cccc}
0 & 0 & -1 & 0 \\
0 & 0 & 0 & -1 \\
1 & 0 & 0 & 0 \\
0 & 1 & 0 & 0
\end{array}\right) \\
& \gamma_{10}=\left(\begin{array}{cccc}
0 & 0 & 1 & 0 \\
0 & 0 & 0 & -1 \\
-1 & 0 & 0 & 0 \\
0 & 1 & 0 & 0
\end{array}\right) \quad \gamma_{11}=-\left(\begin{array}{cccc}
0 & 0 & 1 & 0 \\
0 & 0 & 0 & 1 \\
1 & 0 & 0 & 0 \\
0 & 1 & 0 & 0
\end{array}\right) \\
& \gamma_{12}=\left(\begin{array}{cccc}
-1 & 0 & 0 & 0 \\
0 & -1 & 0 & 0 \\
0 & 0 & 1 & 0 \\
0 & 0 & 0 & 1
\end{array}\right) \quad \gamma_{13}=\left(\begin{array}{cccc}
0 & 0 & 0 & -1 \\
0 & 0 & 1 & 0 \\
0 & 1 & 0 & 0 \\
-1 & 0 & 0 & 0
\end{array}\right) \\
& \gamma_{14}=\left(\begin{array}{cccc}
0 & 0 & 0 & -1 \\
0 & 0 & -1 & 0 \\
0 & 1 & 0 & 0 \\
1 & 0 & 0 & 0
\end{array}\right) \quad \gamma_{15}=\mathbf{1}
\end{aligned}
$$

\section{APPENDIX C: ARBITRARY HAMILTONIANS}

The concept presented above is far more general, as any arbitrary Hamiltonian $H(\Psi)$ may be written as a Taylor series up to second order:

$$
H(\Psi)=H_{0}+\sum_{k} \varepsilon_{k} \Psi_{k}+\frac{1}{2} \sum_{i, k} \Psi_{i} A_{i k} \Psi_{k}+\cdots
$$

From Eqs. (C1) and (14), one finds the following EQOM:

$$
\dot{\Psi}=\gamma_{0} \varepsilon+\gamma_{0} \mathbf{A} \Psi .
$$

Leach formulated a time canonical transformation to transform a time-dependent Hamiltonian of the form given by Eq. (C1) to a different (simpler) form, which we will summarize in the following. Given a symplectic timedependent transformation matrix $\mathbf{R}$, one writes for the spinor $\psi$ in the transformed system:

$$
\psi=\mathbf{R} \Psi+\psi_{0} .
$$

The new Hamiltonian $\tilde{H}$ should have the form

$$
\tilde{H}=\tilde{H}_{0}+\tilde{\varepsilon} \psi+\frac{1}{2} \psi^{T} \tilde{\mathbf{A}} \psi,
$$

and the new EQOM are

$$
\dot{\psi}=\gamma_{0} \tilde{\varepsilon}+\gamma_{0} \tilde{\mathbf{A}} \psi,
$$

or, using Eqs. (C3) and (C2),

$$
\begin{aligned}
\dot{\psi} & =\dot{\mathbf{R}} \Psi+\mathbf{R} \dot{\Psi}+\dot{\psi}_{0} \\
& =\dot{\mathbf{R}} \Psi+\mathbf{R}\left(\gamma_{0} \varepsilon+\gamma_{0} \mathbf{A} \Psi\right)+\dot{\psi}_{0} .
\end{aligned}
$$

Combining Eqs. (C5) and (C6) yields

$$
\begin{aligned}
\dot{\psi}_{0} & =\gamma_{0} \tilde{\mathbf{A}} \psi_{0}-\mathbf{R} \gamma_{0} \boldsymbol{\varepsilon}+\gamma_{0} \tilde{\boldsymbol{\varepsilon}} \\
\dot{\mathbf{R}} & =\gamma_{0} \tilde{\mathbf{A}} \mathbf{R}-\mathbf{R} \gamma_{0} \mathbf{A},
\end{aligned}
$$

where the "coordinate-free and coordinate-dependent parts have been separated" [25].

We are especially interested in a transformation which keeps the forces $\gamma_{0} \mathbf{A}=\gamma_{0} \tilde{\mathbf{A}}$ but sets $\tilde{\varepsilon}=0$ in the new system. One obtains with $\mathbf{F}=\gamma_{0} \mathbf{A}$ :

$$
\dot{\psi}=\mathbf{F} \psi \quad \dot{\psi}_{0}=\mathbf{F} \psi_{0}-\mathbf{R} \gamma_{0} \varepsilon \quad \dot{\mathbf{R}}=\mathbf{F R}-\mathbf{R F} . \quad \text { (C8) }
$$

If one considers the (rather trivial) situation that $\varepsilon=0$, then the conclusion is that any spinor $\psi$ can formally be replaced by $\psi_{0}+\mathbf{R} \psi$ - or more generally: Given a set of vectors $\psi_{k}$ where each vector $\psi_{k}$ fulfills the EQOM

$$
\dot{\psi}_{k}=\mathbf{F} \psi_{k},
$$

then one finds that a superposition of the form

$$
\psi=\sum_{k} \mathbf{R}_{k} \psi_{k}
$$

where the symplectic matrices $\mathbf{R}_{k}$ obey the EQOM

$$
\dot{\mathbf{R}}_{k}=\mathbf{F R}_{k}-\mathbf{R}_{k} \mathbf{F},
$$

also fulfills the EQOM:

$$
\begin{aligned}
\dot{\psi} & =\sum_{k} \mathbf{R}_{k} \dot{\psi}_{k}+\sum_{k} \dot{\mathbf{R}}_{k} \psi_{k} \\
& =\sum_{k} \mathbf{R}_{k} \mathbf{F} \psi_{k}+\sum_{k}\left(\mathbf{F} \mathbf{R}_{k}-\mathbf{R}_{k} \mathbf{F}\right) \psi_{k} \\
& =\mathbf{F} \sum_{k} \mathbf{R}_{k} \psi_{k}=\mathbf{F} \psi
\end{aligned}
$$

\section{APPENDIX D: MULTIPLICATION TABLES OF THE RDMS}

Table IV lists the commutators and Table V the anticommutators of all RDMs. The sum of both is the multiplication table of the group.

\section{APPENDIX E: SECOND MOMENTS AND EXPECTATION VALUES}

In order to obtain the direct relation between the second moments $\sigma_{i j}$ according to Eq. (73) and the expectation values $\tilde{f}_{k}$, we established two new vectors. The first is a vector of second moments: 
$\vec{\sigma}=\left(\sigma_{11}, \sigma_{22}, \sigma_{33}, \sigma_{44}, \sigma_{12}, \sigma_{34}, \sigma_{13}, \sigma_{24}, \sigma_{23}, \sigma_{14}\right)^{T}$,

and the second is the vector $\vec{f}$ of the form

$$
\vec{x}=\left(\tilde{f}_{0}, \tilde{f}_{1}, \tilde{f}_{6}, \tilde{f}_{8}, \tilde{f}_{3}, \tilde{f}_{4}, \tilde{f}_{2}, \tilde{f}_{7}, \tilde{f}_{5}, \tilde{f}_{9}\right)^{T} .
$$

Ordered in this form, the equation

$$
\vec{\sigma}=\mathbf{T} \vec{x}
$$

holds for a block-diagonal matrix $\mathbf{T}$ :

$$
\mathbf{T}=\frac{1}{2}\left(\begin{array}{cccccccccc}
1 & -1 & 1 & 1 & & & & & & \\
1 & 1 & -1 & 1 & & & & & & \\
1 & 1 & 1 & -1 & & & & & & \\
1 & -1 & -1 & -1 & & & & & & \\
& & & & 1 & 1 & & & & \\
& & & & 1 & -1 & & & & \\
& & & & & & 1 & 1 & & \\
& & & & & & -1 & 1 & & \\
& & & & & & & & -1 & -1 \\
& & & & & & & & -1 & 1
\end{array}\right)
$$

Note that the ordering is chosen such that the first six (last four) elements of $\vec{x}$ correspond to expectation values of even (odd) RDMs. The inverse $\mathbf{T}^{-1}$ has the same block structure.

[1] G. Dattoli, C. Mari, L. Mezi, and A. Torre, Nucl. Instrum. Methods Phys. Res., Sect. A 321, 447 (1992).

[2] G. Dattoli, C. Mari, C. Ronsivalle, and A. Torre, Nuovo Cimento 107, 179 (1992).

[3] H. Qin and R.C. Davidson, Phys. Plasmas 16, 050705 (2009).

[4] H. Qin and R.C. Davidson, in Proceedings of the 23rd Particle Accelerator Conference, Vancouver, Canada, 2009 (IEEE, Piscataway, NJ, 2009).
[5] H. Qin, R. C. Davidson, M. Chung, J. J. Barnard, and T. F. Wang, in Proceedings of 2011 Particle Accelerator Conference, New York, NY, USA, 2011 [http://accelconf .web.cern.ch/AccelConf/PAC2011/papers/tuocn5.pdf].

[6] V. A. Lebedev and S. A. Bogacz, JINST 5, P10010 (2010).

[7] Yun Luo, Phys. Rev. ST Accel. Beams 7, 124001 (2004).

[8] L.C. Teng, NAL-Report No. FN-229, 1971.

[9] D. A. Edwards and L. C. Teng, IEEE Trans. Nucl. Sci. 20, 885 (1973).

[10] K. L. Brown and R. V. Servranckx, Part. Accel. 36, 121 (1991).

[11] Andrej Wolski, Phys. Rev. ST Accel. Beams 9, 024001 (2006).

[12] C. Baumgarten, arXiv:1109.1996v1.

[13] Ettore Majorana, Nuovo Cimento 14, 171 (1937) [Ettore Majorana Scientific Papers 10, 201 (2006)].

[14] F. Wilczek, Nature Phys. 5, 614 (2009).

[15] G. Dattoli, C. Mari, M. Richetta, and A. Torre, Il Nuovo Cimento B 107, 269 (1992).

[16] Susumu Okubo, Math. Jap. 41, 59 (1995) [http://arxiv.org/ abs/hep-th/9408165v1].

[17] K. Scharnhorst, J. Math. Phys. (N.Y.) 40, 3616 (1999).

[18] V.I. Arnold, Mathematical Methods of Classical Mechanics (Springer, New York, 2010), 2nd ed.

[19] R. Talman, Geometric Mechanics (Wiley-VCH Weinheim, Germany, 2007), 2nd ed.

[20] Frank Hinterberger, Physik der Teilchenbeschleuniger (Springer, Heidelberg, 2008), Vol. 2.

[21] E. D. Courant and H. S. Snyder, Ann. Phys. (Leipzig) 3, 1 (1958); Ann. Phys. (N.Y.) 281, 360 (2000).

[22] H. Goldstein, C. Poole, and J. Safko; Classical Mechanics (Addison-Wesley, San Francisco, 2002), 3rd ed.

[23] R. H. Good, Jr., Rev. Mod. Phys. 27, 187 (1955).

[24] K. L. Brown, SLAC-Report No. 75, 1982.

[25] P. G. Leach, J. Math. Phys. (N.Y.) 18, 1608 (1977).

[26] L. L. Foldy and S. A. Wouthuysen, Phys. Rev. 78, 29 (1950).

[27] D. Hestenes, J. Math. Phys. (N.Y.) 8, 798 (1967).

[28] D. Hestenes, J. Math. Phys. (N.Y.) 16, 556 (1975).

[29] Y. Nambu, Phys. Rev. D 7, 2405 (1973). 